NASA Contractor Report 187603

ICASE Report No. 91-57

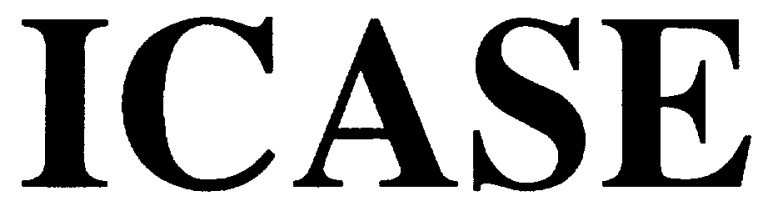

MULTIGRID FOR HYPERSONIC VISCOUS TWOAND THREE-DIMENSIONAL FLOWS

E. Turkel

R. C. Swanson

V. N. Vatsa

J. A. White

Contract No. NAS1-18605

July 1991

Institute for Computer Applications in Science and Engineering NASA Langley Research Center

Hampton, Virginia 23665-5225

Operated by the Universities Space Research Association

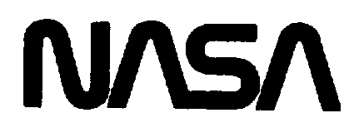

National Aeronautics and

Space Adminislration

Langley Research Center

Hampton, Virginia 23665-5225 
$\sim$ 


\title{
Multigrid for Hypersonic Viscous Two- and Three-Dimensional Flows
}

\author{
E. Turkel * \\ Tel-Aviv University \\ Tel-Aviv 69978, Israel \\ and \\ Institute for Computer Applications in Science and Engineering \\ Hampton, VA 23665 \\ R. C. Swanson \\ and \\ V. N. Vatsa \\ NASA Langley Research Center \\ Hampton, VA 23665 \\ J. A. White \\ Analytical Services and Materials, Inc. \\ Hampton, VA 23665
}

\begin{abstract}
We consider the use of a multigrid method with central differencing to solve the Navier-Stokes equations for hypersonic flows. The time-dependent form of the equations is integrated with an explicit Runge-Kutta scheme accelerated by local time stepping and implicit residual smoothing. Variable coefficients are developed for the implicit process that remove the diffusion limit on the time step, producing significant improvement in convergence. A numerical dissipation formulation that provides good shock-capturing capability for hypersonic flows is presented. This formulation is shown to be a crucial aspect of the multigrid method. Solutions are given for two-dimensional viscous flow over a NACA 0012 airfoil and three-dimensional viscous flow over a blunt biconic.
\end{abstract}

*This research was partially supported by the National Aeronautics and Space Administration under NASA Contract No. NAS1-18605 while the first author was in residence at the Institute for Computer Applications in Science and Engineering (ICASE) NASA Langley Research Center, Hampton, VA 23665. 
- 


\section{Introduction}

At the present time there is a strong interest in high-speed flight vehicles. Some examples of these vehicles are the high-speed civil transport (HSCT) and the hypersonic flight configurations being considered for the National Aero-Space Plane (NASP). In the case of the NASP, one encounters complex high Mach number phenomena and interactions, which can involve strong shock and expansion waves, in not only the flow over the vehicle, but also in the flow through the engines, where chemical reactions occur. An effective design method for such vehicles will obviously require both detailed experimental data as well as flexible, efficient, and accurate computational techniques. Robust prediction methods (i.e., those that can be applied on a routine basis) are not currently available for hypersonic flows. However, due to the significant progress during the last decade in the development of effective algorithms for subsonic and transonic flows, there are a number of opportunities for constructing improved schemes for high-speed flows.

One powerful approach for the numerical solution of partial differential equations, which has been successfully applied to fluid flow problems, is multigrid. Multigrid methods were first developed for elliptic equations. These were later extended to hyperbolic equations such as the time-dependent fluid dynamic equations for subsonic and transonic flow $[1,2,3,4,5]$. Even for transonic cases, the steady state can retain many of the properties of an elliptic equation when the region of supersonic flow is limited. We shall show that with proper care the multigrid method still works for hypersonic flow. Gustafsson and Lotstedt [6] have pointed out that hyperbolic multigrid works by two different processes. For the long waves, the advection process is most important and multigrid achieves its efficiency by allowing the use of larger time steps on coarser grids. Hence, it is important that the smoother use large time steps. However, for the shorter waves, dissipation is more important and the efficiency of multigrid is based on principles similar to that for elliptic equations.

Several investigators have applied multigrid to high-speed flows with varying degrees of success. For example, in [7] the Euler equations are solved with and without chemistry using the Roe scheme for spatial discretization and the ADI scheme for time marching. A factor of four decrease in computational time was obtained with the multigrid method for a simple one-dimensional nozzle flow (exit Mach number of about 3.7). A calculation for a Mach 22 wedge flow showed the basic scheme to be noticeably faster than the multigrid scheme. One notable conclusion of this work was that the performance of the multigrid is driven by the fluid dynamics and not the chemistry (at least for the case of simple reaction models). The high level of performance and widespread application of multigrid algorithms with central differencing and an explicit multistage time-stepping scheme have provided strong encouragement to make them work for hypersonic problems. An initial effort [8] to apply this type of algorithm resulted in numerical difficulties that prevented the calculation of two-dimensional flows (i.e., blunt body and wedge type) with a Mach number higher than about 7 . In order to compute such flows, a low CourantFriedrichs-Lewy (CFL) number was required. Thus four and five stage schemes were not practical, since there is substantial deterioration in the high frequency damping of the scheme due to the large reduction in the CFL number. The CFL restriction reduced the potential of the scheme as a viscous flow solver. More recently an algorithm utilizing a semicoarsening technique, a symmetric TVD formulation, and a three stage Runge-Kutta scheme [9] was proposed and used to compute high Reynolds number (laminar) Mach 10 flow over an airfoil at 10 degrees angle of attack. A good resolution of the bow shock 
wave and a reasonable convergence rate were obtained. The method of semicoarsening considered required a much more complicated cycle strategy than that employed with standard multigrid methods. In addition, it appears to be somewhat cumbersome to implement in three dimensions.

It is our contention that standard multigrid techniques can be used in conjunction with central differencing for hypersonic flows. To do this one must ensure that the basic algorithm exhibits good damping of high frequencies, on both the fine and coarse meshes, in the neighborhood of strong discontinuities. It becomes more difficult to eliminate these high frequency oscillations as the Mach number increases, since the jumps across shocks become larger. Thus a considerable part of the following discussion will concentrate on the smoothing algorithm. The fundamental features of the multigrid process (i.e., Full Approximation Storage scheme, grid transfer operators, fixed cycle strategy) are fairly standard. Other aspects, such as type of coarse grid correction scheme and procedure for smoothing of coarse grid corrections, found crucial in the present work will be emphasized. In this paper we consider a Runge-Kutta scheme [10] as the smoother for the multigrid method. Central differences for spatial approximations are augmented by an artificial viscosity based on TVD principles [11]. Several changes are made to the numerical algorithm so that a converged solution can be obtained for high-speed flows. Initially, we describe the basic Runge-Kutta method for the centraldifference scheme with the numerical viscosity. We discuss an implicit procedure for accelerating the convergence of the basic scheme. This procedure removes the diffusion limit on the time step, which can be quite severe at hypersonic speeds. We then apply the multigrid method to solve both two- and three-dimensional viscous flows.

\section{Basic Scheme}

The basic elements of the scalar dissipation model considered in this paper were first introduced by Jameson, Schmidt, and Turkel [10] in conjunction with Runge-Kutta explicit schemes. The spatial discretization is based on central differences with an additional artificial viscosity. This algorithm has been used by many investigators to solve the Euler equations numerically for a wide range of fluid dynamic applications. The same type of spatial discretization has been applied to alternating direction implicit (ADI) schemes [12] and LU factored implicit schemes [13]. In this section the basic scheme is briefly reviewed. Since the elements of the two-dimensional and three-dimensional schemes are essentially the same, we will take, for the purpose of simplicity, a twodimensional point of view in describing the basic scheme.

Consider the Euler equations in the form

$$
W_{t}+f_{x}+g_{y}=0
$$

where the four-component vector of conserved variables

$$
W=\left[\begin{array}{llll}
\rho & \rho u & \rho v & \rho E
\end{array}\right]^{T},
$$

and $f, g$ are the corresponding flux vectors. The quantity $\rho$ is the density, $u$ and $v$ are the Cartesian velocity components, and $E$ is the specific total internal energy. The independent variables are time $t$ and Cartesian coordinates $(x, y)$. If $(1)$ is transformed to arbitrary curvilinear coordinates $\xi=\xi(x, y)$ and $\eta=\eta(x, y)$, then we obtain

$$
\left(J^{-1} W\right)_{t}+F_{\xi}+G_{\eta}=0
$$


where $J^{-1}$ is the inverse transformation Jacobian, and

$$
F=f y_{\eta}-g x_{\eta}, \quad G=g x_{\xi}-f y_{\xi} .
$$

In a cell-centered, finite-volume method, (1) is integrated over an elemental volume in the discretized computational domain, and $J^{-1}$ is identified as the volume of the cell. Equation (3) can also be written as

$$
J^{-1} W_{t}+A W_{\xi}+B W_{\eta}=0
$$

where $A$ and $B$ are the flux Jacobian matrices defined by $A=\partial F / \partial W$ and $B=\partial G / \partial W$.

To advance the scheme in time we use a multistage scheme. A typical step of a Runge-Kutta approximation to (3) is

$$
W^{(k)}=W^{(0)}-\alpha_{k} \frac{\Delta t}{J^{-1}}\left[D_{\xi} F^{(k-1)}+D_{\eta} G^{(k-1)}-A D\right],
$$

where $D_{\xi}$ and $D_{\eta}$ are spatial differencing operators, and $A D$ represents the artificial dissipation terms. The derivatives of the fluxes are approximated by central differences. Hence, we need to evaluate $F$ at $\left(i+\frac{1}{2}\right)$. For the physical diffusive fluxes the approximations follow directly. Since the dependent variables are given at $(i, j)$ we need to average them to calculate the convective flux at the cell face. This averaging can be done in several ways. In the original scheme the conservative variables were averaged to find the variables at the cell face and then the flux was evaluated. Moreover, in two dimensions five quantities were averaged, the four conservative variables and $\rho E+p$. This was done because enthalpy damping was used. Alternatively one can average density, momentum, and total enthalpy. This might be more accurate for the Euler equations since the total enthalpy is constant in the steady state. An alternative to this procedure is to average the fluxes rather than the dependent variables, where the fluxes are defined as for (3) and the transformation derivatives are evaluated at the cell interface. Averaging the fluxes allows for a more accurate representation of the Rankine-Hugoniot jump conditions in one dimension. In this paper we average the fluxes but have not seen much difference between the various approaches.

The dissipation terms are a blending of second and fourth differences. That is,

$$
A D=\left(D_{\xi}^{2}+D_{\eta}^{2}-D_{\xi}^{4}-D_{\eta}^{4}\right) W
$$

where

$$
\begin{gathered}
D_{\xi}^{2} W=\nabla_{\xi}\left[\left(\lambda_{\xi_{i+\frac{1}{2}, j}} \epsilon_{i+\frac{1}{2}, j}^{(2)}\right) \Delta_{\xi}\right] W_{i, j}, \\
D_{\xi}^{4} W=\nabla_{\xi}\left[\left(\lambda_{\xi_{i+\frac{1}{2}, j}} \epsilon_{i+\frac{1}{2}, j}^{(4)}\right) \Delta_{\xi} \nabla_{\xi} \Delta_{\xi}\right] W_{i, j},
\end{gathered}
$$

and $\Delta_{\xi}, \nabla_{\xi}$ are the standard forward and backward difference operators, respectively, associated with the $\xi$ direction. The variable scaling factor $\lambda$ is chosen as

$$
\lambda_{\xi_{i+\frac{1}{2}, j}}=\frac{1}{2}\left[\left(\lambda_{\xi}\right)_{i, j}+\left(\lambda_{\xi}\right)_{i+1, j}\right]
$$

where $\lambda_{\xi}$ is proportional to the spectral radius of the matrix $A$. The coefficients $\epsilon^{(2)}$ and $\epsilon^{(4)}$ are adapted to the flow and are defined as follows:

$$
\epsilon_{i+\frac{1}{2}, j}^{(2)}=\kappa^{(2)} \max \left(\nu_{i-1, j}, \nu_{i, j}, \nu_{i+1, j}, \nu_{i+2, j}\right)
$$




$$
\begin{aligned}
\nu_{i, j} & =\left|\frac{p_{i+1, j}-2 p_{i, j}+p_{i-1, j}}{p_{i+1, j}+2 p_{i, j}+p_{i-1, j}}\right|, \\
\epsilon_{i+\frac{1}{2}, j}^{(4)} & =\max \left[0,\left(\kappa^{(4)}-\epsilon_{i+\frac{1}{2}, j}^{(2)}\right)\right],
\end{aligned}
$$

where $p$ is the pressure, and the quantities $\kappa^{(2)}$ and $\kappa^{(4)}$ are constants to be specified. The operators for the $\eta$ direction are defined in a similar manner.

The second-difference dissipation term is nonlinear. Its purpose is to introduce an entropy-like condition and to suppress oscillations in the neighborhood of shocks. This term is small in the smooth portion of the flow field. The fourth-difference dissipation term is basically linear and is included to damp high-frequency modes and allow the scheme to approach a steady state. Only this term affects the linear stability of the scheme. Near shocks it is reduced to zero. For high-speed flows, the switch (10) is not very good and does not allow the multigrid to converge. Instead we consider a TVD variation of the switch [11] given by

$$
\nu_{i, j}=\frac{\left|p_{i+1, j}-2 p_{i, j}+p_{i-1, j}\right|}{\left|p_{i+1, j}-p_{i, j}\right|+\left|p_{i, j}-p_{i-1, j}\right|+\epsilon}, \quad \kappa^{(2)}=1 / 2 .
$$

With this change and the factor $1 / 2$ in front of the second-difference dissipation term, the scalar equation becomes first-order upwind near shocks. In the case of the original $\nu$, we find that $\nu \simeq 0.05$ near shock waves in transonic flows. The parameter $\epsilon$ must be chosen carefully to prevent the switch from being activated by noise. In fact, we found it useful to take an average of the two versions for $\nu$. Hence, we use

$$
\nu_{i, j}=\frac{\left|p_{i+1, j}-2 p_{i, j}+p_{i-1, j}\right|}{(1-\omega)\left(P_{T V D}\right)_{i, j}+\omega P_{i, j}}
$$

where

$$
\begin{aligned}
\left(P_{T V D}\right)_{i, j} & =\left|p_{i+1, j}-p_{i, j}\right|+\left|p_{i, j}-p_{i-1, j}\right| \\
P_{i, j} & =p_{i+1, j}+2 p_{i, j}+p_{i-1, j}
\end{aligned}
$$

and $\omega$ is taken to be $1 / 2$. We now no longer have a free parameter for the seconddifference dissipation.

Several other changes were made to the scheme in addition to the change to a TVD switch. In the original algorithm, the artificial viscosity for the energy equation was based on the total enthalpy rather than the total internal energy. For high-speed flows we base the artificial viscosity on the total internal energy so that in each equation the basic dependent variable is also used in the artificial viscosity. This is more in line with upwind schemes. This has previously been used in central-difference schemes [14]. The algorithm no longer preserves a constant total enthalpy in the steady state (as the Euler equations do), but enthalpy damping is not useful for supersonic flows. In most cases the difference between the two approaches is small with each approach having its advantages. The original version seems to give slightly sharper shocks, while the other one appears to make the scheme more robust.

The form of the dissipation model of the basic or driving scheme is usually modified for the coarse grids in the multigrid process. A constant coefficient, second-difference dissipation is not only less expensive computationally but also generally provides adequate 
smoothing properties. For high-speed flows we find it necessary to append a nonlinear dissipation to the usual one that depends on the modified switching function of (12). We also need to increase the constant coefficient on a coarse mesh from the standard value of $1 / 16$ to a value of $1 / 4$.

In order for the scheme to be stable it is necessary to restrict the time step. Both a convection limit as well as a diffusion limit must be taken into account in general. As shown in [15] the actual time step $\left(\Delta t_{a c t}\right)$, based on a sufficient condition for stability, is determined as follows:

$$
\Delta t_{a c t} \leq N\left[\bar{\lambda}_{\xi}+\bar{\lambda}_{\eta}+d \bar{\lambda}_{v i s c o u s}\right]^{-1}
$$

where $N$ is taken to be the allowable CFL number, and the constant $d$ is 4 . The spectral radii $\bar{\lambda}_{\xi}$ and $\bar{\lambda}_{\eta}$ of the Jacobian matrices $\tilde{A}$ and $\tilde{B}$, where the tilde denotes that the matrix is multiplied by the transformation Jacobian $J$, are given by

$$
\begin{aligned}
& \bar{\lambda}_{\xi}=\left|u \xi_{x}+v \xi_{y}\right|+c \sqrt{\xi_{x}^{2}+\xi_{y}^{2}}, \\
& \bar{\lambda}_{\eta}=\left|v \eta_{y}+u \eta_{x}\right|+c \sqrt{\eta_{x}^{2}+\eta_{y}^{2}} .
\end{aligned}
$$

For the thin-layer Navier-Stokes equations,

$$
\bar{\lambda}_{\text {viscous }}=\frac{\gamma \mu}{\operatorname{Pr} \rho}\left(\eta_{x}^{2}+\eta_{y}^{2}\right)
$$

where $\gamma$ is the specific heat ratio, and $\operatorname{Pr}$ is the Prandtl number.

We now consider how the contributions to (14) behave near the body as the inflow Mach number is increased. We assume that the variables are normalized so that the density and pressure are 1 at inflow. In these nondimensional units the viscous terms are multiplied by $\frac{\sqrt{\gamma} M_{\infty}}{R e}$. In the boundary layer the velocity goes to zero and so $\lambda_{\eta}$ basically behaves as the sound speed c. If $\mu=T=p / \rho$, then

$$
\frac{\bar{\lambda}_{\text {viscous }}}{\bar{\lambda}_{\eta}} \sim \frac{M_{\infty} \sqrt{p}}{\rho^{\frac{3}{2}} \operatorname{Re} e_{\infty} P r} .
$$

We next consider the ratios $p_{t_{2}} / p_{1}$ and $\rho_{t_{2}} / \rho_{1}$ for adiabatic flow [16]. As $M_{\infty}$ becomes larger $\mathrm{p}$ behaves like $6 M_{\infty}^{2} / 5$ while $\rho$ approaches 6 . So as $M_{\infty}$ becomes larger

$$
\frac{\bar{\lambda}_{\text {viscous }}}{\bar{\lambda}_{\eta}} \sim M_{\infty}^{2}
$$

Thus, the diffusion limit on the time step is quite significant for high Mach numbers.

For all flow calculations in this paper a five stage Runge-Kutta scheme with a weighted evaluation, as detailed in [17], of the dissipation terms on the first, third, and fifth stages is used. The time step is reduced near shocks by including a term that depends on $\nu_{i, j}$. The reduction is constructed so that there is a CFL number of 1 when $\nu=1$. It serves to reduce the magnitude of the change in the solution near the shock wave, which exhibits strong nonlinear behavior. 


\section{Implicit Residual Smoothing}

A mathematical step can be included in the basic time-stepping scheme to extend the local stability. This procedure is called implicit smoothing (or averaging) of the residuals. Previously, variable coefficients for this implicit process have been introduced by Martinelli [2] and Swanson and Turkel [18]. In addition, an explicit multidimensional derivation of such coefficients is considered in [19]. These variable coefficients have proven to be quite reliable in extending the stability limit of the Runge-Kutta scheme by a factor of two. However, their development is based on hyperbolic considerations only, and they do not eliminate the need for a diffusion factor in the time step. As indicated in the preceding section and we emphasize here, this factor can even dominate the time step determination in the case of hypersonic flow. In this section we will briefly discuss the smoothing coefficients of [18] and present their straightforward extension to three dimensions. Then we will develop smoothing coefficients that allow the removal of the diffusion limit, resulting in a $\Delta t$ which depends only on the spectral radii of the flux Jacobian matrices.

For multidimensional problems, the residual smoothing can be applied in the form

$$
\left[\prod_{l}\left(I-\beta_{l} \nabla_{l} \Delta_{l}\right)\right] \overline{\mathcal{R}}_{i, j}^{(m)}=\mathcal{R}_{i, j}^{(m)} \quad, \quad l=\xi, \eta, \zeta
$$

where the residual $\mathcal{R}_{i, j}^{(m)}$ is defined by

$$
\mathcal{R}_{i, j}^{(m)}=\alpha_{m} \frac{\Delta t_{i, j}}{\Omega_{i, j}}\left[\mathcal{L}_{C} W_{i, j}^{(m-1)}+\mathcal{L}_{D} W_{i, j}^{(0)}-A D^{(m)}\right],
$$

and computed in the Runge-Kutta stage $m$. The quantity $A D^{(m)}$ is the total artificial dissipation at stage $m$, and $\overline{\mathcal{R}}_{i, j}^{(m)}$ is the final residual at stage $m$ after the sequence of smoothings involving each coordinate direction. The difference operators $\mathcal{L}_{C}$ and $\mathcal{L}_{D}$ are associated with the convection and physical diffusion terms. To derive the maximum stability extension for the hyperbolic problem, the implicit procedure is applied after each stage of the Runge-Kutta scheme. The coefficients are functions of the spectral radii $\lambda_{\xi}, \lambda_{\eta}$, and $\lambda_{\zeta}$. In two dimensions they are written as follows:

$$
\begin{aligned}
& \beta_{\xi}=\max \left\{\frac{1}{4}\left[\left(\frac{N}{N^{*}} \frac{1}{1+\psi r_{\eta \xi}}\right)^{2}-1\right], 0\right\}, \\
& \beta_{\eta}=\max \left\{\frac{1}{4}\left[\left(\frac{N}{N^{*}} \frac{1}{1+\psi r_{\eta \xi}^{-1}}\right)^{2}-1\right], 0\right\},
\end{aligned}
$$

where the ratio $r_{\eta \xi}=\lambda_{\eta} / \lambda_{\xi}$, and the quantity $N / N^{*}$ is the ratio of the CFL number of the smoothed scheme to that of the basic explicit scheme (usually having a value of 2 ). In hypersonic flow applications we found it necessary for $N^{*}$ to be 3.25 , rather than the value of 3.75 used for transonic computations. From a linear stability analysis, the scheme with these coefficients is stable for all mesh cell aspect ratios when the parameter 
$\psi \approx 0.125$ and $N / N^{*}$ is sufficiently large. The practical limitation on the Courant number is due to the requirement for effective high frequency damping. For large $N / N^{*}$, the high frequency damping of the scheme vanishes. The variable coefficients are functions of the local mesh cell aspect ratio, and thus the smoothing process is not activated in a coordinate direction where it is not needed. This is important for best possible convergence.

The formulas of (17) and (18) can be easily extended to three dimensions. Moreover, we define

$$
\beta_{l}=\max \left\{\frac{1}{4}\left[\left(\frac{N}{N^{*}} \Phi_{l}\right)^{2}-1\right], 0\right\}, \quad l=\xi, \eta, \zeta
$$

where

$$
\begin{aligned}
& \Phi_{\xi}=\frac{1}{1+\psi_{3-D}\left(r_{\eta \xi}+r_{\zeta \xi}\right)}, \\
& \Phi_{\eta}=\frac{1}{1+\psi_{3-D}\left(r_{\eta \xi}^{-1}+r_{\zeta \eta}\right)}, \\
& \Phi_{\zeta}=\frac{1}{1+\psi_{3-D}\left(r_{\zeta \xi}^{-1}+r_{\zeta \eta}^{-1}\right)} .
\end{aligned}
$$

Once again $r$ represents a ratio of characteristic speeds. A typical value for $\psi_{3-D}$ is 0.0625 .

We now show how one can utilize these coefficients as the basis for new coefficients that will remove the diffusion limit on the time step. Consider the scalar diffusion equation

$$
\frac{\partial w}{\partial t}=\mu \frac{\partial^{2} w}{\partial y^{2}}
$$

If we approximate the spatial derivative of (20) with a central difference and take a Fourier transform, we obtain

$$
\Delta t \frac{d \hat{w}}{d t}=Z \hat{w}^{n}
$$

where the caret indicates a transformed quantity, and the Fourier symbol

$$
Z=-2 \lambda_{d}(1-\cos \theta)
$$

with $\lambda_{d}=\Delta t \mu / \Delta y^{2}$. If implicit residual smoothing is applied, then

$$
Z=\frac{-2 \lambda_{d}(1-\cos \theta)}{1+2 \beta_{d}(1-\cos \theta)}
$$

A sufficient condition for stability is given by

$$
|Z| \leq N_{d}^{*} \quad \text { for all } \theta
$$

where $N_{d}^{*}$ is the diffusion number of the unsmoothed scheme. It then follows that the smoothing coefficient $\beta_{d}$ is given by

$$
\beta_{d} \geq \frac{1}{4}\left[\frac{N_{d}}{N_{d}^{*}}-1\right]=\frac{1}{4}\left[\frac{\Delta t_{d}}{\Delta t_{d}^{*}}-1\right]
$$


where $N_{d}=4 \lambda_{d}$. This implicit smoothing coefficient has a linear dependence on the diffusion number ratio. In the case of the pure hyperbolic problem, one obtains a quadratic dependence with the CFL number ratio. Thus we must determine a way to combine these results to obtain a coefficient that is valid for an equation with both convection and diffusion effects.

Suppose we consider the thin-layer form of the two-dimensional Navier-Stokes equations, then one could simply use the smoothing coefficient of (17) in the streamwise-like $(\xi)$ direction. One would anticipate that a possible formulation for the normal $\eta$ direction would depend on a diffusion type $\beta$ near the surface and a convection type $\beta$ when the viscous effects are no longer important. Notice the dependency in (25) on the ratio of the actual $\Delta t$ to the $\Delta t$ of the basic explicit scheme. So we define

$$
\left(\beta_{d}\right)_{\eta}=\frac{1}{4}\left[\frac{\Delta t_{a c t}}{\left(\Delta t_{d}\right)_{\eta}}-1\right]
$$

Since we want to remove the diffusion limit, the actual $\Delta t$ must depend only on $\lambda_{\xi}$ and $\lambda_{\eta}$. Thus we rewrite (26) as

$$
\left(\beta_{d}\right)_{\eta}=\frac{1}{4}\left[C_{1} \frac{\left(\lambda_{d}\right)_{\eta}}{\lambda_{\xi}+\lambda_{\eta}}-1\right],
$$

which we can approximate simply as

$$
\left(\beta_{d}\right)_{\eta}=\frac{1}{4} \bar{C}_{1} \frac{\left(\lambda_{d}\right)_{\eta}}{\lambda_{\xi}+\lambda_{\eta}} .
$$

It does not appear to matter whether one uses the form of (27) or that of (28), provided the constant is defined properly. Here we have elected to use the simpler form of (28), which has also been considered by Radespiel and Kroll [20]. From numerical experiments we found that a satisfactory value for $\bar{C}_{1}$ is 10 .

The variable coefficient of (28) cannot generally be used by itself. For example, in an airfoil flow, $\left(\beta_{d}\right)_{\eta}$ goes to zero too fast at the leading edge, resulting in a zero value in the inviscid region. We have overcome this difficulty by calculating $\beta_{\eta}$ as

$$
\beta_{\eta}=\max \left(\left(\beta_{d}\right)_{\eta},\left(\beta_{c}\right)_{\eta}\right)
$$

where $\left(\beta_{c}\right)_{\eta}$ is defined by (18). In the Results section of this paper we consider Mach 10 and Mach 20 turbulent flow over an airfoil. The removal of the diffusion limit allowed the residual in each case to be reduced at least an additional order of magnitude in 200 multigrid cycles.

\section{Multigrid Method}

As indicated earlier the salient features of the multigrid method considered here are fairly standard. We apply the Full A pproximation Storage (FAS) scheme of Brandt [21] to define the equivalent fine grid problem on a coarse grid. The grid transfer operators for the solution, residual, and coarse grid corrections are those introduced by Jameson [1]. In order to execute the multigrid strategy we employ a fixed W-type cycle with two sweeps on each coarse grid. To provide a well conditioned starting solution for the fine 
mesh a Full Multigrid (FMG) method is used. The FMG is analogous to grid sequencing, except that multigrid cycles are performed on each coarse grid.

Some of the additional elements of the multigrid method are not necessarily standard. A smoothing of the coarse grid corrections being transferred to the finest grid was found to be beneficial in transonic computations [17]. The smoothing was accomplished with the implicit residual smoothing mentioned previously and a constant coefficient $\beta \approx 0.1$. This smoothing of the residuals on the way to finer meshes is crucial for the convergence of the multigrid for hypersonic flows. Such a process acts to reduce high frequency oscillations caused by the interpolation. Hence, it becomes especially important near strong shocks, where nonphysical upstream influence can occur. It should be emphasized that choosing the smoothing parameter too large can slow convergence. Another important element for high Mach number $(M \geq 10)$ flows is the coarse grid correction scheme. That is, the physical viscous terms should also be computed on the coarse meshes. Difficulties with applying a turbulence model on a coarse grid can be avoided by interpolating the turbulent viscosity from the values on the finest grid.

\section{Boundary Conditions and Initialization}

At a solid surface (wall) boundary the no-slip condition is enforced. The wall pressure is set to the value at the first interior solution point, and thus, a reduced normal momentum equation is satisfied. An adiabatic wall is assumed. In a finite-volume formulation, this amounts to treating the Cartesian velocity components as antisymmetric functions and the temperature as a symmetric function with respect to the wall. For each of the physical problems considered the Mach number at the inflow boundary exceeds 1.0. Consequently, the dependent variables are specified at this boundary according to the flow conditions. At any outflow boundary, we apply simple extrapolation of the components of the solution vector. In general, for hypersonic flows, numerical difficulties are experienced at the start of a calculation if the discrete flow field is initialized with free-stream conditions. To avoid these difficulties we apply the following procedure. The Mach number of the flow is set to a lower value than the required one. Then the Mach number is gradually increased over a few hundred time steps until the desired flow conditions are obtained. This Mach number ramping is only done on the coarsest mesh in the FMG sequence.

\section{Results}

We consider the following two test cases: (1) two-dimensional turbulent flow about the NACA 0012 airfoil, and (2) three-dimensional turbulent flow about a blunt biconic. For these cases we found a significant sensitivity to the grid. With a grid suitable for transonic flows (i.e., a standard C-type mesh), difficulties in convergence were experienced at high Mach numbers. Furthermore, the usual mesh density for airfoil flows yielded a poor resolution of the flow field. The grids which were generated for these examples all follow in a general sense the bow shock. The bow shock is not aligned with the grid, but as a minimum, the angle between the bow shock and the grid is not too large. We also found it important to control the normal stretching rate of the grid in the inviscid region of the flow field.

For the first case we consider turbulent flow (a Reynolds number $R e_{c}=10^{7}$ ) about the NACA 0012 airfoil at two different Mach numbers. In all the calculations, transition 
was assumed to occur at the 20 percent chord location. This ensured that the algebraic turbulence model of Baldwin and Lomax [22] did not determine an unrealistic length scale in the vicinity of the leading edge due to the presence of the shock. The grids for the computations were generated with the method of [23]. The fine grid, consisting of $320 \times 64$ cells, is displayed in figure 1 . In the streamwise direction, the grid is clustered at the leading and trailing edges of the airfoil, with a tangential spacing of approximately $1 \times 10^{-3}$ chords. There is clustering in the normal direction adjacent to the surface, so as to provide adequate resolution of the turbulent boundary layer. The minimum normal spacing occurs at the airfoil leading edge and is $1 \times 10^{-5}$ chords. To obtain reasonable shock definition everywhere, there is weak normal clustering in the inviscid region.

Figures 2 and 3 show the Mach number and pressure contours, respectively, when $M_{\infty}=10$. There is fairly good resolution of the bow shock, and the contours are smooth. The pressure contours delineate the expected fish tail shock in the wake region. Surface pressure and skin-friction distributions for this case are presented in figure 4 . In addition, velocity profiles at the midchord and 95 percent chord locations are shown in figure 5. The same results computed on a $160 \times 32$ mesh, which is a proper subset of the $320 \times 64$ mesh, are also included in these figures. One can clearly see that there is fairly good agreement between the predictions on the two meshes. Thus, additional mesh refinement will serve primarily to improve the downstream resolution of the bow shock. In figure 6 the variations of the Mach number and pressure along the stagnation streamline are shown. Comparing the pressure jump and the stagnation pressure with one-dimensional theory, we find the differences are less than 2 percent. With the cellcentered, finite-volume scheme, one should keep in mind that the computational points do not lie precisely along the stagnation streamline.

Convergence histories for the Mach 10 case are given in figure 7 . On the finest mesh the logarithm of the residual of the continuity equation is reduced 7.5 orders of magnitude in 200 multigrid cycles. This corresponds to an average rate of reduction of 0.917. The calculation required less than 6 minutes on a Cray II computer. It should be emphasized that for engineering accuracy (i.e., residual reduced by 3 orders) the finest mesh calculation required less than 3 minutes of CPU time. Note that when engineering accuracy is achieved, there is no appreciable improvement in the viscous solution accuracy by further residual reduction.

In figures 8-11 results for $M_{\infty}=20$ and $R e_{c}=10^{7}$ are presented. Again there is fairly good representation of the bow shock, and the Mach and pressure contours are smooth. Here also there is less than a 2 percent difference between the computed pressure jump and the stagnation pressure determined along the stagnation streamline and the values from one-dimensional theory. As indicated in figure 12, the logarithm of the residual is reduced nearly 5 orders in 200 cycles on the finest mesh. There is a slowdown in the asymptotic convergence rate for this case. At this point the precise reason for such behavior is not clear.

We now consider the three-dimensional case. A mesh consisting of $128 \times 96 \times 24$ cells was used for the blunt biconic. In figure 13, a two-dimensional slice of the mesh is depicted. This was constructed using an algebraic mesh generator. A tangent hyperbolic distribution function was used in the normal direction to obtain an acceptable mesh in both the viscous and inviscid regions. For this turbulent flow case, $M_{\infty}=6, \alpha=5$ degrees, and the Reynolds number based on nose diameter is $2.89 \times 10^{5}$. Figures 14 and $15(\mathrm{a})$ - 15(b) show Mach number and pressure contours, respectively, in the symmetry 
plane. One sees the small standoff distance of the shock at the nose and how the shock remains relatively close to the geometry downstream. The shock surface is delineated in the three-dimensional view of pressure contours given in figure 15(c). A comparison is made in figure 16 between computed pressure distributions, for the windward and leeward planes, and the experimental data of [24]. There is generally good agreement with the data. In figure 17 the convergence history is presented for this computation. The logarithm of the residual has been reduced about 5.5 orders of magnitude in about 565 work units, which roughly corresponds to 350 multigrid cycles on the finest mesh. For engineering accuracy, 85 cycles are required on the finest mesh, and the CPU time is about 30 minutes.

\section{Concluding Remarks}

A multigrid method with central differencing has been successfully applied to the solution of hypersonic viscous flows. An explicit five stage Runge-Kutta scheme has been used as a smoother in solving the time-dependent, thin-layer Navier-Stokes equations. In this paper, considerable emphasis has been focussed on the dissipative characteristics of the driving scheme for the multigrid process. The presence of strong shocks has required the introduction of a switching function for the numerical dissipation based on TVD principles. This nonlinear switching function is also required for the coarse grid dissipation model. The importance of the physical diffusion limit on the time step for computing hypersonic flows has been discussed. By introducing appropriate variable coefficients for implicit residual smoothing, we have removed the diffusion limit and have improved the convergence significantly.

Numerical solutions have been obtained for two- and three-dimensional hypersonic turbulent flows. The agreement between predictions for flow over a blunt biconic compare well with experimental data. Engineering accuracy has been obtained rapidly in all computations, requiring less than $3 \mathrm{CPU}$ minutes on a Cray II for two-dimensional cases and about $30 \mathrm{CPU}$ minutes for a three-dimensional case. 


\section{References}

[1] Jameson, A. and Baker, T. J., Multigrid Solution of the Euler Equations for Aircraft Configurations, AIAA Paper 84-0093, 1984.

[2] Martinelli, L., Calculations of Viscous Flows with a Multigrid Method, Ph. D. Dissertation, MAE Department, Princeton University, Oct. 1987.

[3] Swanson, R. C. and Turkel, E., Artificial Dissipation and Central Difference Schemes for the Euler and Navier-Stokes Equations, AIAA Paper 87-1107, AIAA 8th Computational Fluid Dynamics Conference, Honolulu, Hawaii, 1987.

[4] Vatsa, V. N. and Wedan, B. W., Development of an Efficient Multigrid Code for 3-D Navier-Stokes Equations, AIAA Paper 89-1791, 1989.

[5] Radespiel, R., Rossow C., and Swanson, R. C., An Efficient Cell-Vertex Multigrid Scheme for the Three-Dimensional Navier-Stokes Equations, AIAA Paper 89-1953, 1989.

[6] Gustafsson, B. and Lotstedt, P., Analysis of the Multigrid Method Applied to First Order Systems, Proccedings Fourth Copper Mountain Conference on Multigrid Methods, ed. by J. Mandel et. al., SIAM, Philadelphia, 1990.

[7] Slomski, J., Anderson, J., and Gorski, J., Effectiveness of Multigrid in Accelerating Convergence of Multidimensional Flows in Chemical Nonequilibrium, AIAA Paper 90-1575, June 1990.

[8] Kroll, N., Radespiel, R., and Rossow C.-C., Experiences with Explicit Time-Stepping Schemes for Supersonic Flow Fields, Proceedings of Eighth GAMM Conference on Numerical Methods in Fluid Dynamics, Delft, The Netherlands, Sept. 27-29, 1989.

[9] Radespiel, R. and Kroll, N., A Multigrid Scheme with Semicoarsening for Accurate Computations of Viscous Flows 12th International Conference on Numerical Methods in Fluid Dynamics, University of Oxford, July 1990.

[10] Jameson, A., Schmidt, W., and Turkel, E., Numerical Solutions of the Euler Equations by Finite Volume Methods Using Runge-Kutta Time-Stepping Schemes, AIAA Paper 81-1259, 1981.

[11] Swanson, R. C. and Turkel, E., On Central-Difference and Upwind Schemes, ICASE Report 90-44, 1990, submitted to Journal of Comput. Physics.

[12] Pulliam, T. H., Artificial Dissipation for the Euler Equations, AIAA Journal, Vol.24, 1986, pp. 1931-1940.

[13] Jameson, A. and Yoon, S., Lower-Upper Implicit Schemes with Multiple Grids for the Euler Equations, AIAA Journal, Vol. 25, 1987, pp. 929-935.

[14] Caughey, D. A. and Turkel, E., Effects of Numerical Dissipation on Finite-Volume Solutions to Compressible Flow Problems, AIAA Paper 88-0621, 1988. 
[15] Swanson, R. C., Turkel, E., and White, J. A., An Effective Multigrid Method for High-Speed Flows, Fifth Copper Mountain Conference on Multigrid Methods, Colorado, March 31 - April 5, 1991.

[16] National Advisory Committe for Aeronautics, Report 1135, Equations, Tables and Charts for Compressible Flow, Ames Research Staff, 1953.

[17] Swanson, R. C. and Radespiel, R., Cell Centered and Cell Vertex Multigrid Schemes for the Navier-Stokes Equations, AIAA Journal, Vol. 29, 1991, pp. 697-703.

[18] Swanson, R. C. and Turkel, E., Multistage Schemes with Multigrid for the Euler and Navier-Stokes Equations, NASA Technical Paper, 1991 (to be published)

[19] Wigton, L. B. and Swanson, R. C., Variable Coefficient Implicit Residual Smoothing, 12 th International Conference on Numerical Methods in Fluid Dynamics, University of Oxford, England, July 1990.

[20] Radespiel, R. and Kroll, N., Multigrid Schemes with Semicoarsening for Accurate Computations of Hypersonic Viscous Flows, Tagungsband 7, DGLRFachsymposium "Strermungen mit Ablersung, "Aachen, Nov. 7-9, 1990.

[21] Brandt, A., Multi-Level Adaptive Solutions to Boundary-Value Problems, Vol. 31, No. 138, April 1977, pp. 333-390.

[22] Baldwin, B. S. and Lomax, H., Thin Layer Approximation and Algebraic Model for Separated Turbulent Flows, AIAA Paper 78-257, 1978.

[23] White, J. A., Elliptic Grid Generation with Orthogonality and Spacing Control on an Arbitrary Number of Boundaries, AIAA Paper 90-1568, June 1990.

[24] Miller, C. G. and Gnoffo, P. A., Pressure Distributions and Shock Shapes for $12.84^{\circ} /$ $7^{\circ}$ On-Axis and Bent-Nose Biconics in Air at Mach 6, NASA TM 83222, 1981. 


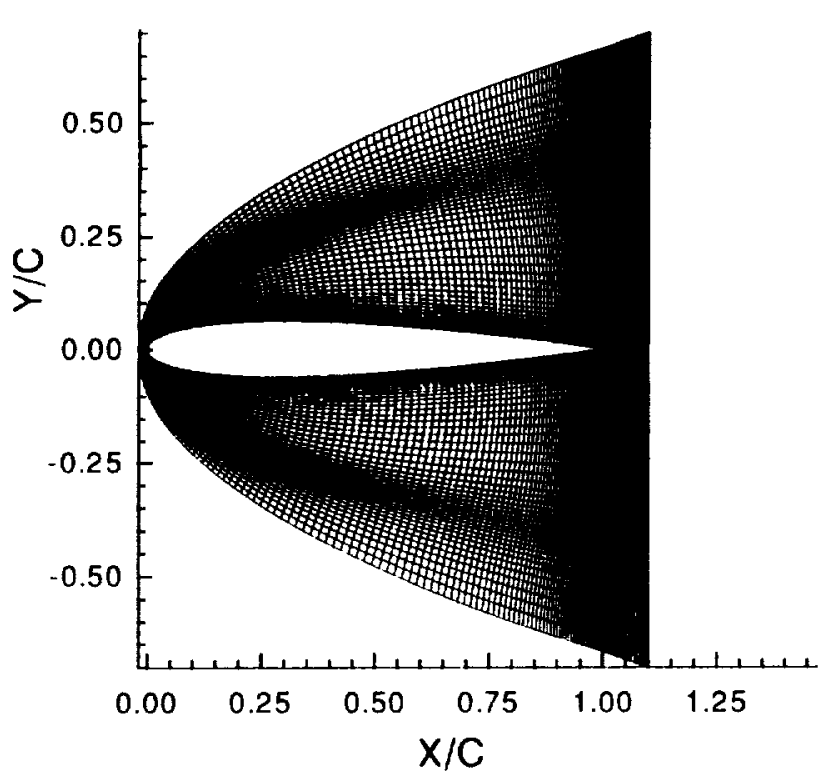

(a) $320 \times 64$ grid

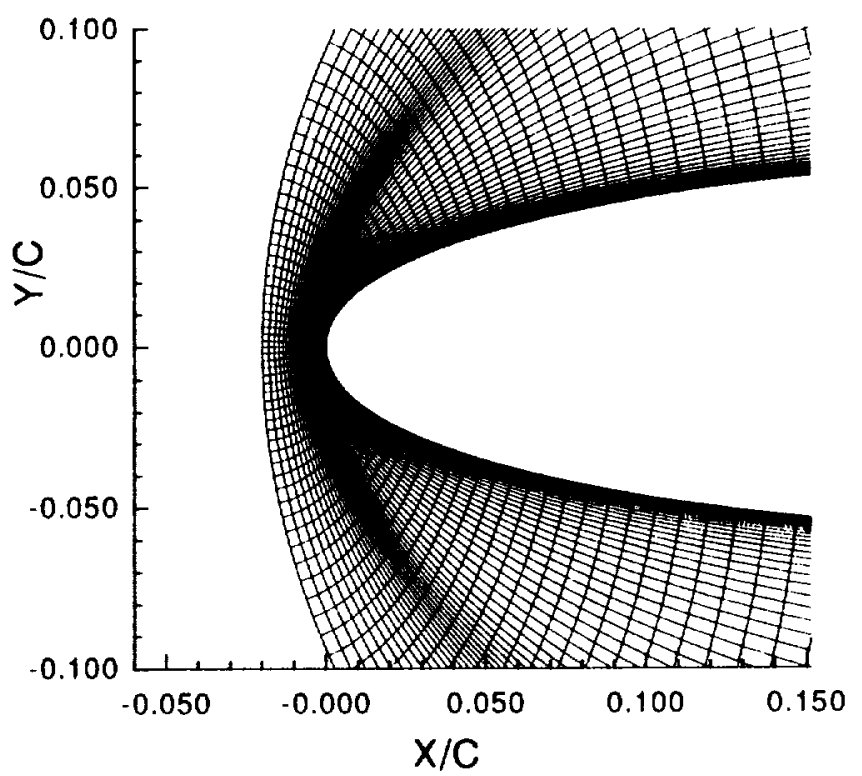

(b) Blowup of leading edge region

Figure 1 A grid for turbulent flow over NACA 0012 airfoil

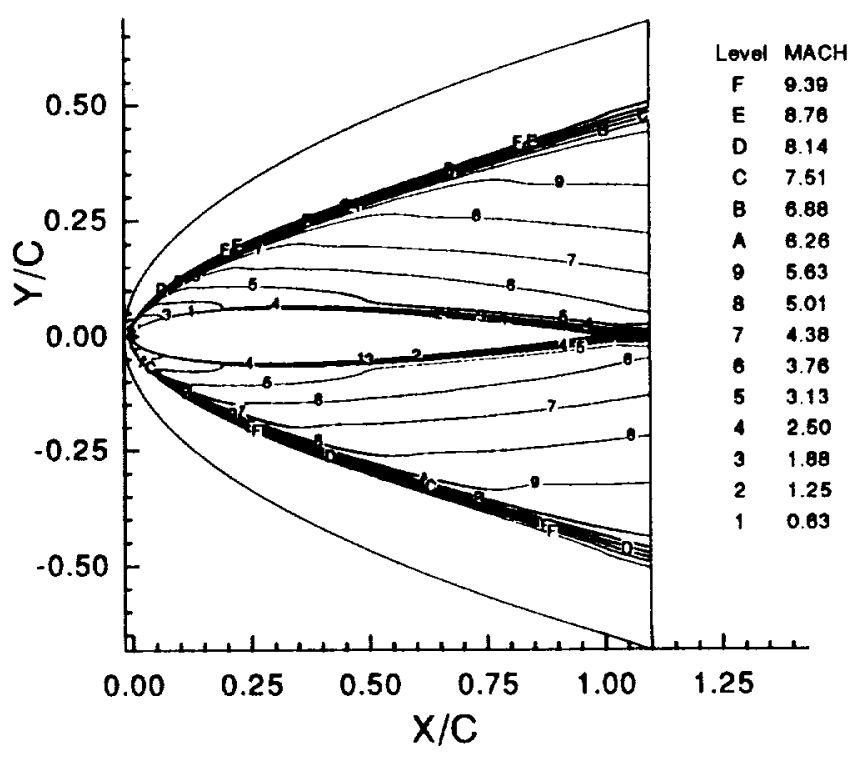

(a) Complete airfoil

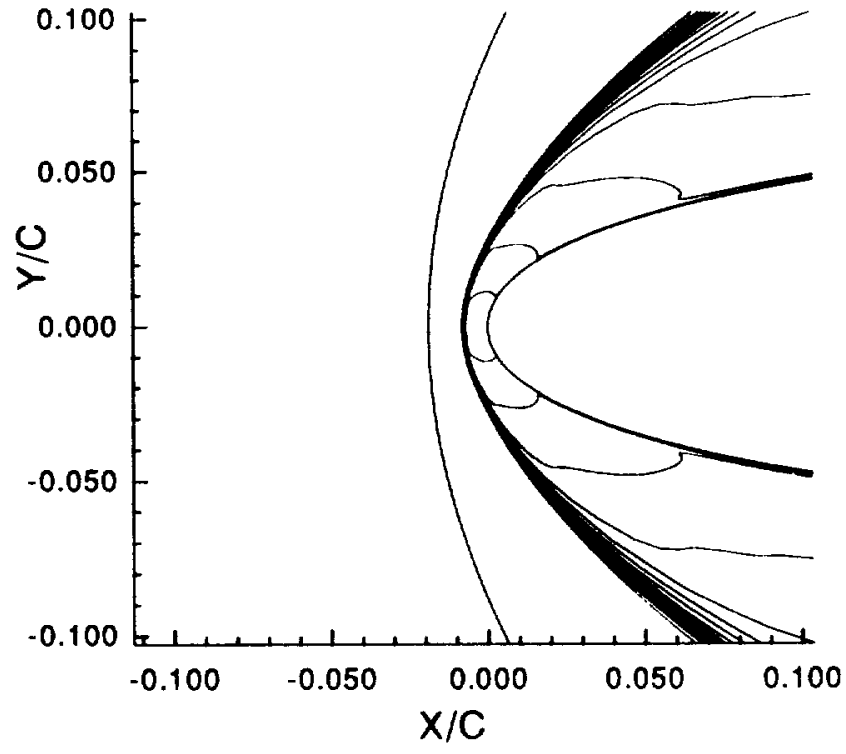

(b) Leading edge region

Figure 2 Mach number contours for Mach 10 turbulent flow over NACA 0012 airfoil $\left(\alpha=0 \mathrm{deg}, R e_{c}=10^{7}\right)$ 


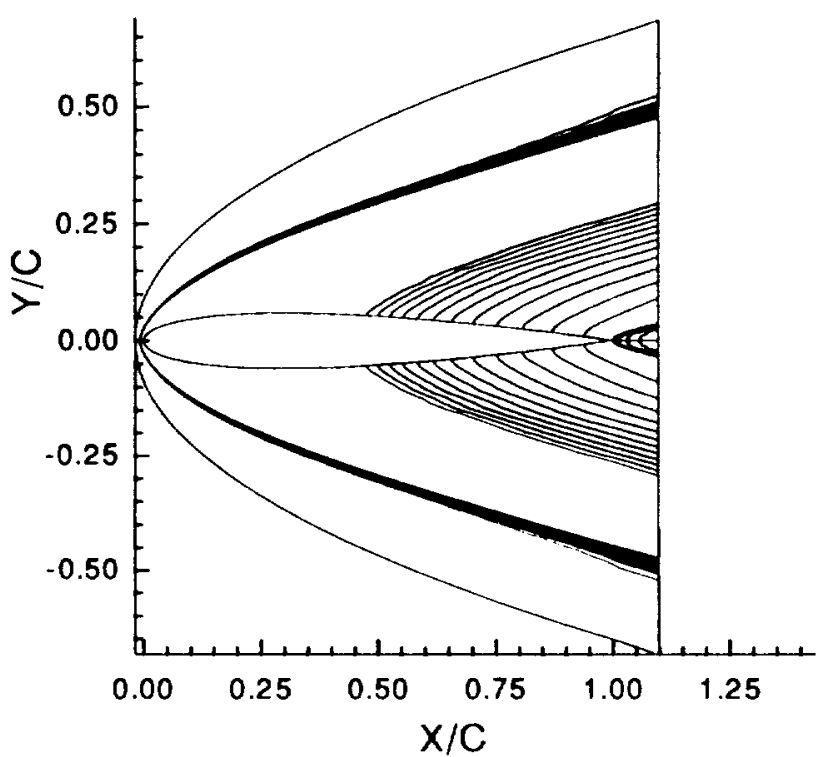

(a) Complete airfoil

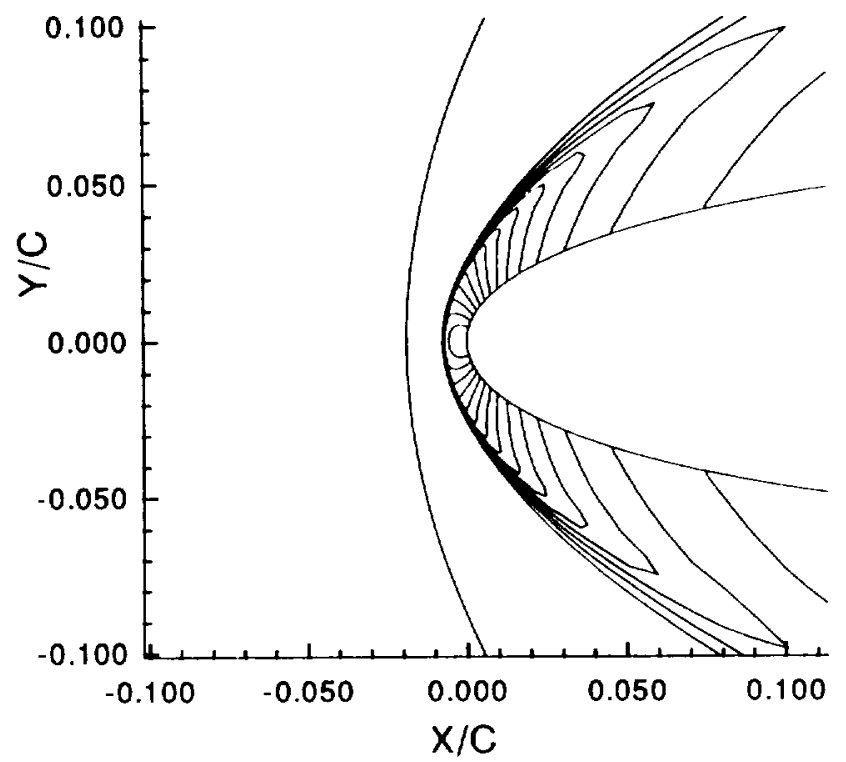

(b) Leading edge region

Figure 3 Pressure contours for Mach 10 turbulent flow over NACA 0012 airfoil $\left(\alpha=0 \mathrm{deg}, R e_{c}=10^{7}\right)$

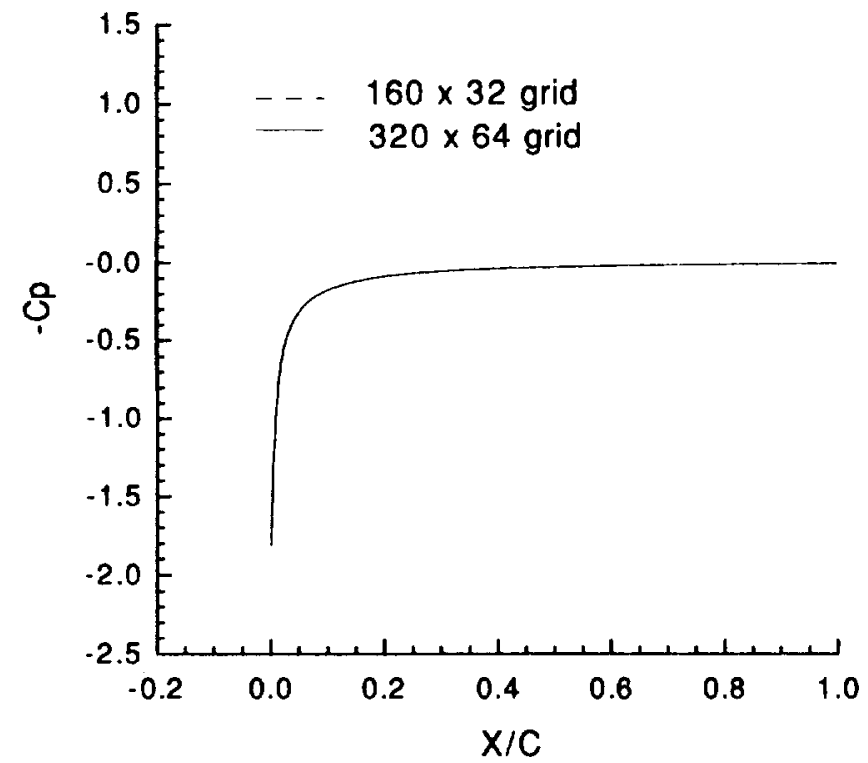

(a) Surface pressure coefficients

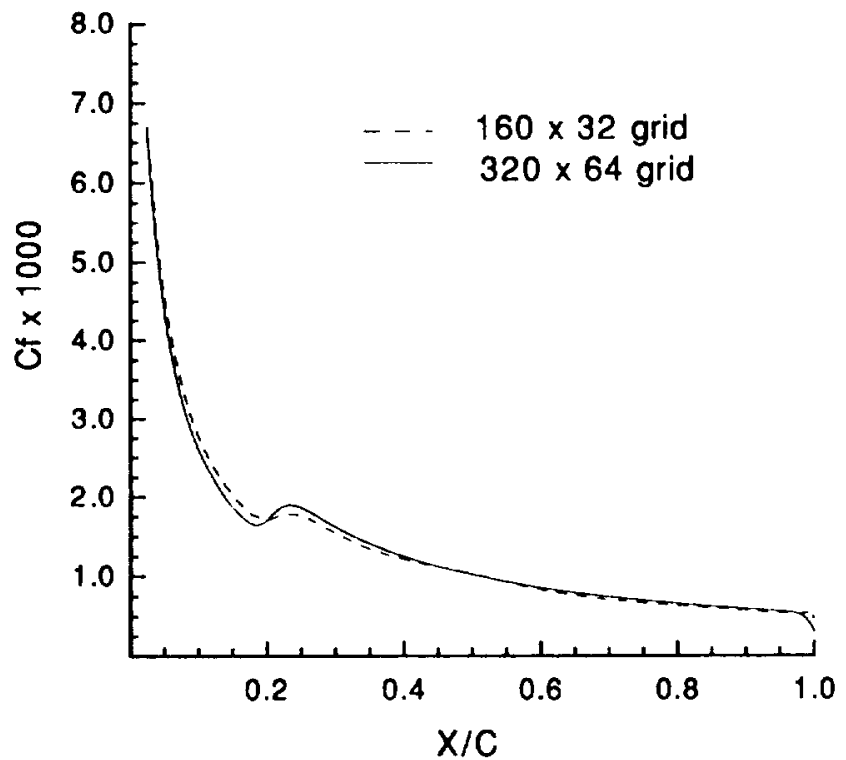

(b) Surface skin-friction coefficients

Figure 4 Surface pressure and skin-friction coefficients for Mach 10 turbulent flow over NACA 0012 airfoil $\left(\alpha=0 \mathrm{deg}, R e_{\mathfrak{c}}=10^{7}\right)$ 


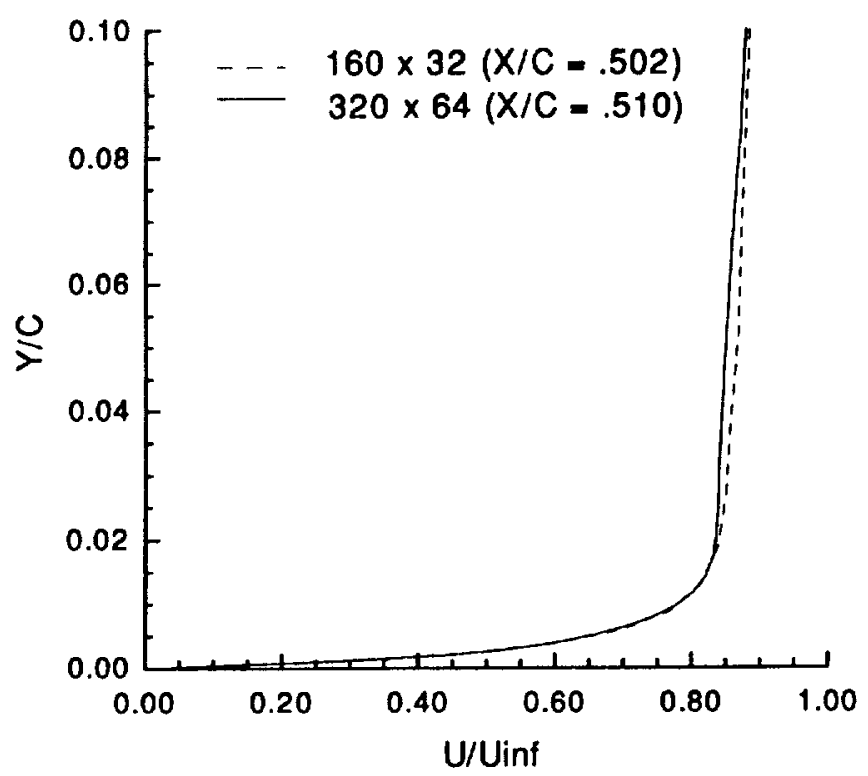

(a) $\mathrm{X} / \mathrm{C}=0.5$

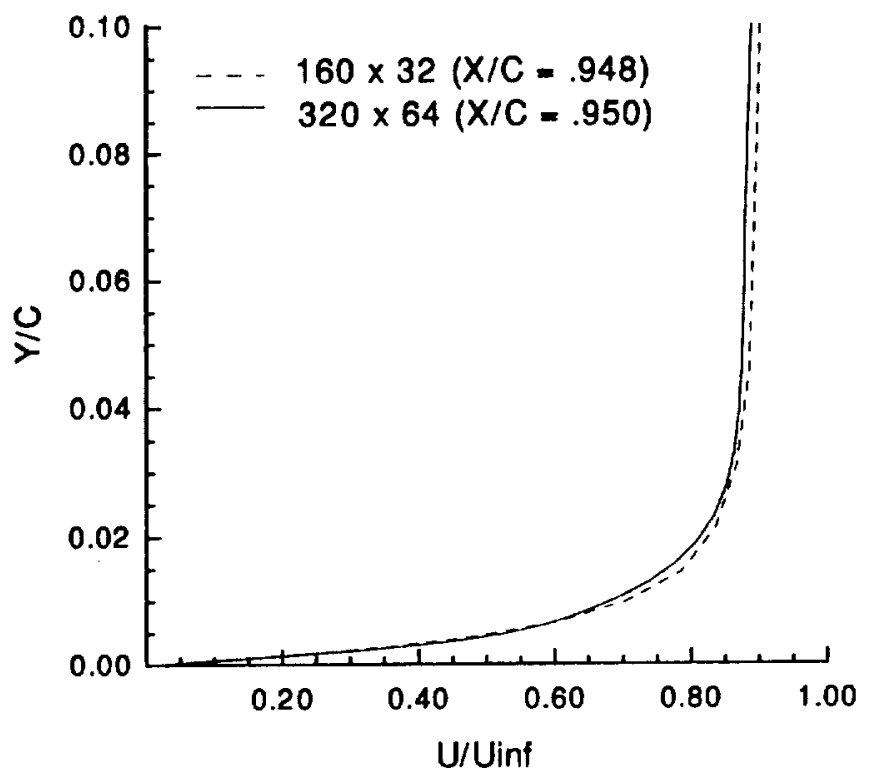

(b) $\mathrm{X} / \mathrm{C}=0.95$

Figure 5 Velocity profiles for Mach 10 turbulent flow over NACA 0012 airfoil $\left(\alpha=0 \mathrm{deg}, R e_{c}=10^{7}\right)$

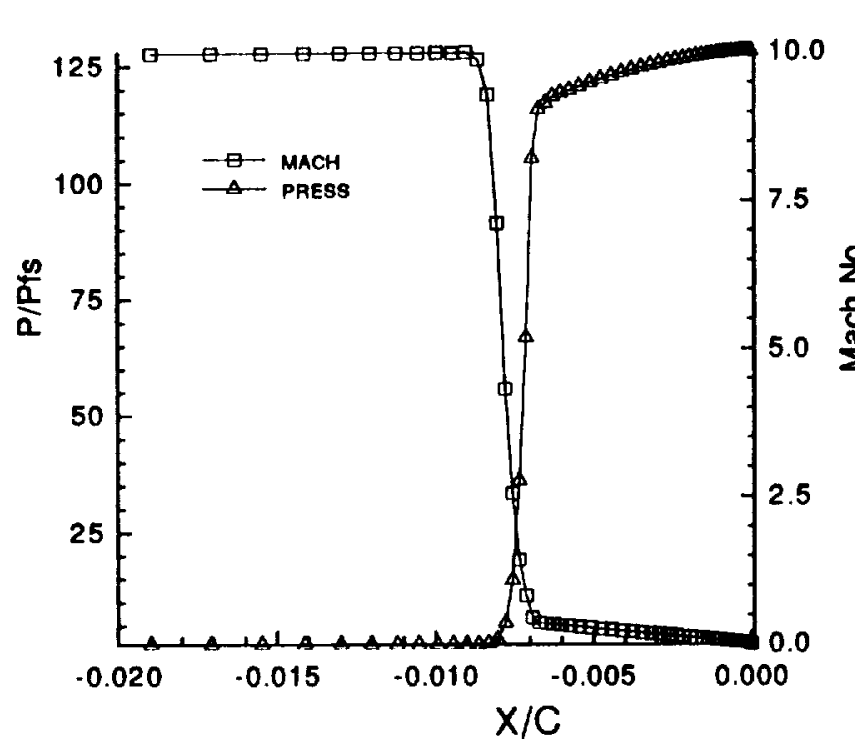

Figure 6 Pressure and Mach number variations along stagnation streamline for Mach 10 turbulent flow (NACA 0012 airfoil, $\alpha=0 \mathrm{deg}, R e_{c}=10^{7}$ )

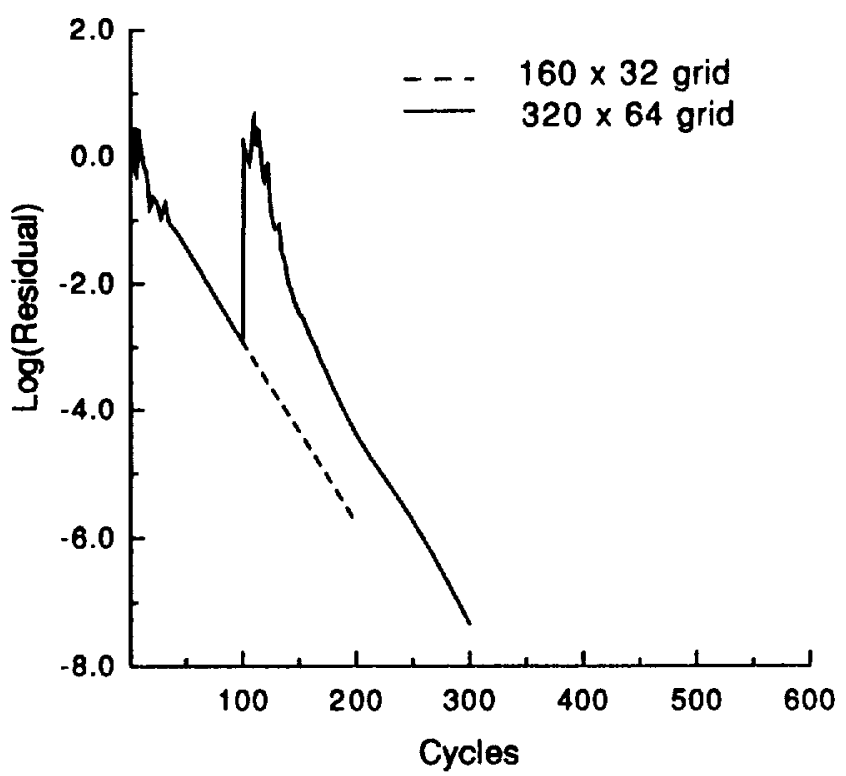

Figure 7 Convergence histories for Mach 10 turbulent flow (NACA 0012 airfoil, $\alpha=0 \mathrm{deg}, R e_{c}=10^{7}$ ) 


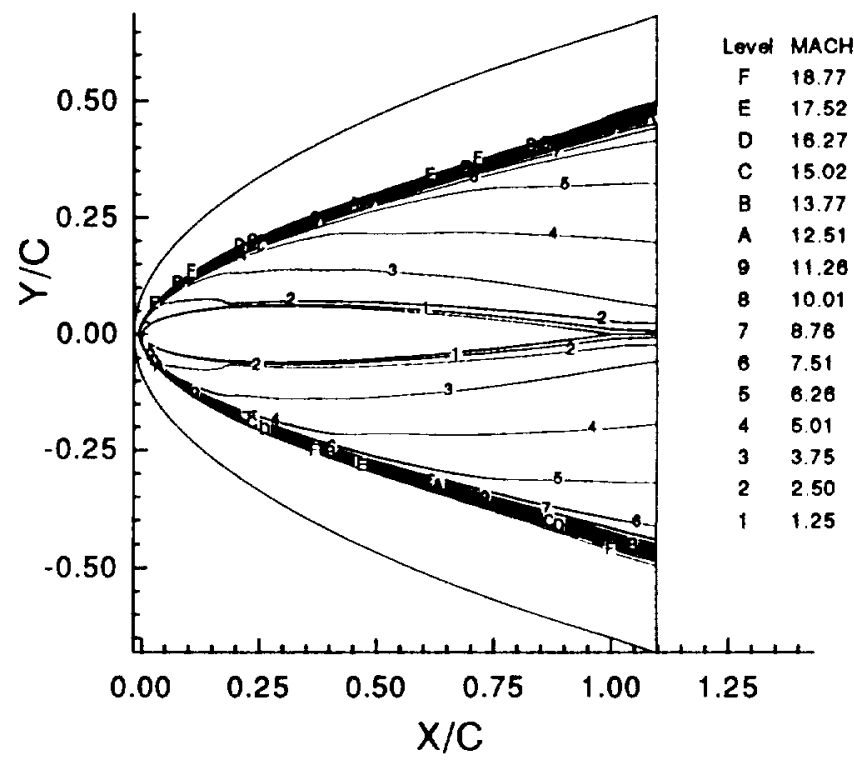

(a) Complete airfoil

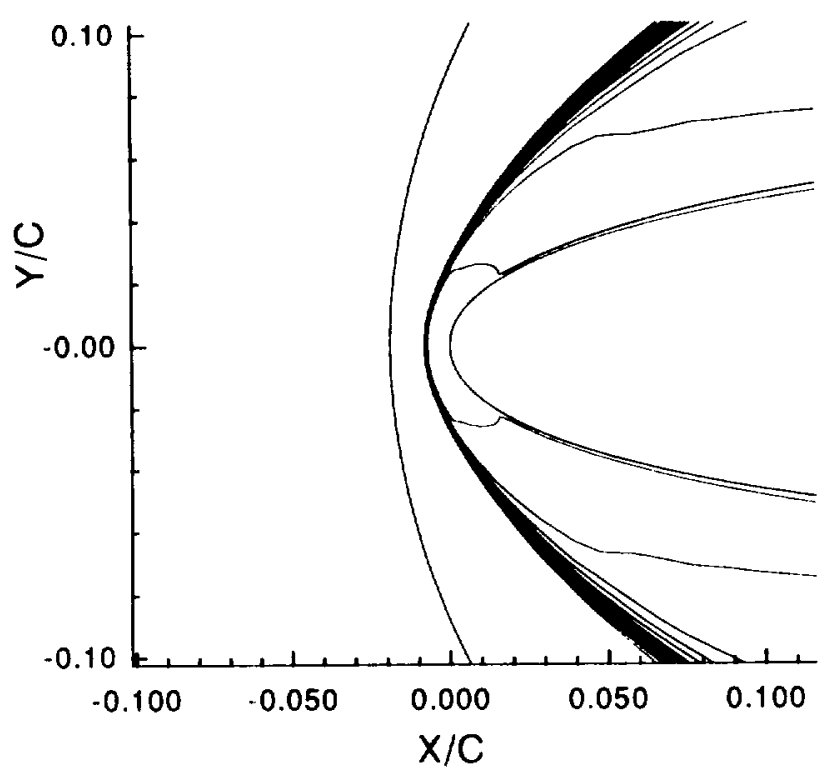

(b) Leading edge region

Figure 8 Mach number contours for Mach 20 turbulent flow over NACA 0012 airfoil $\left(\alpha=0 \mathrm{deg}, R e_{c}=10^{7}\right)$

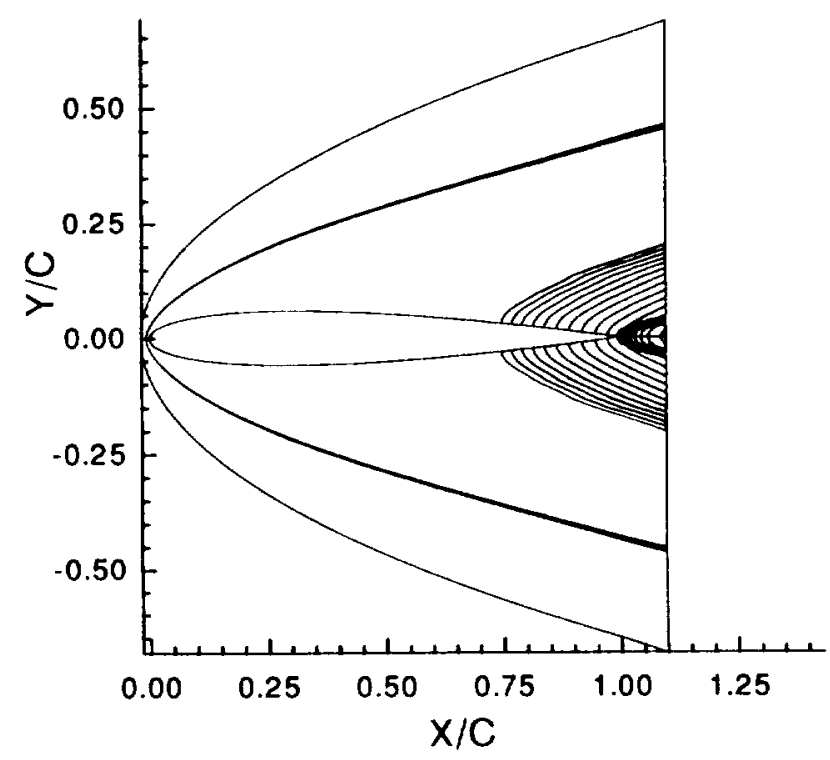

(a) Complete airfoil

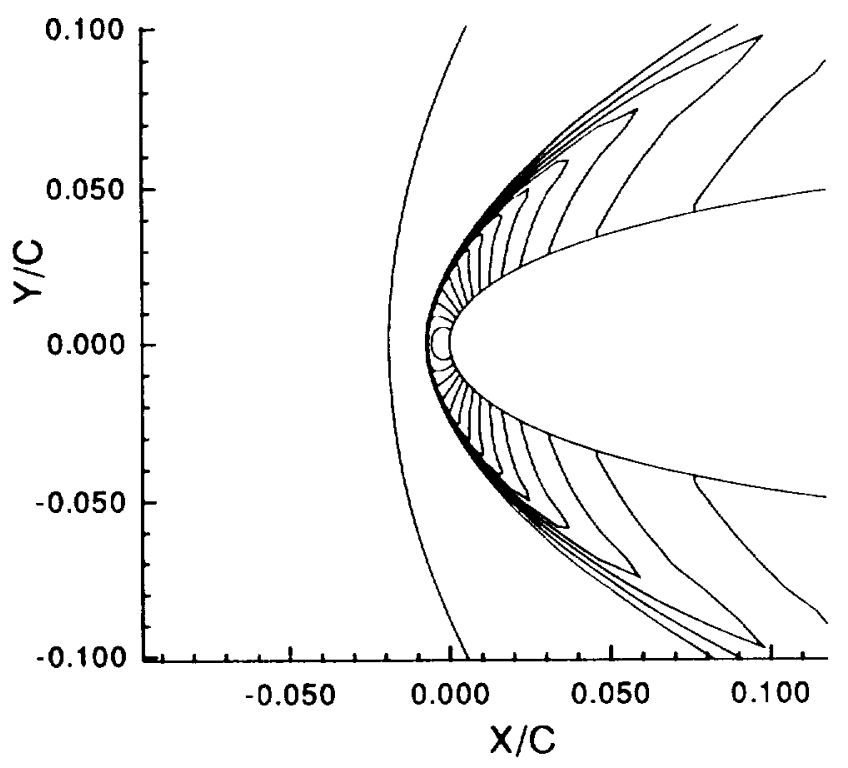

(b) Leading edge region

Figure 9 Pressure contours for Mach 20 turbulent flow over NACA 0012 airfoil $\left(\alpha=0 \mathrm{deg}, R e_{c}=10^{7}\right)$ 


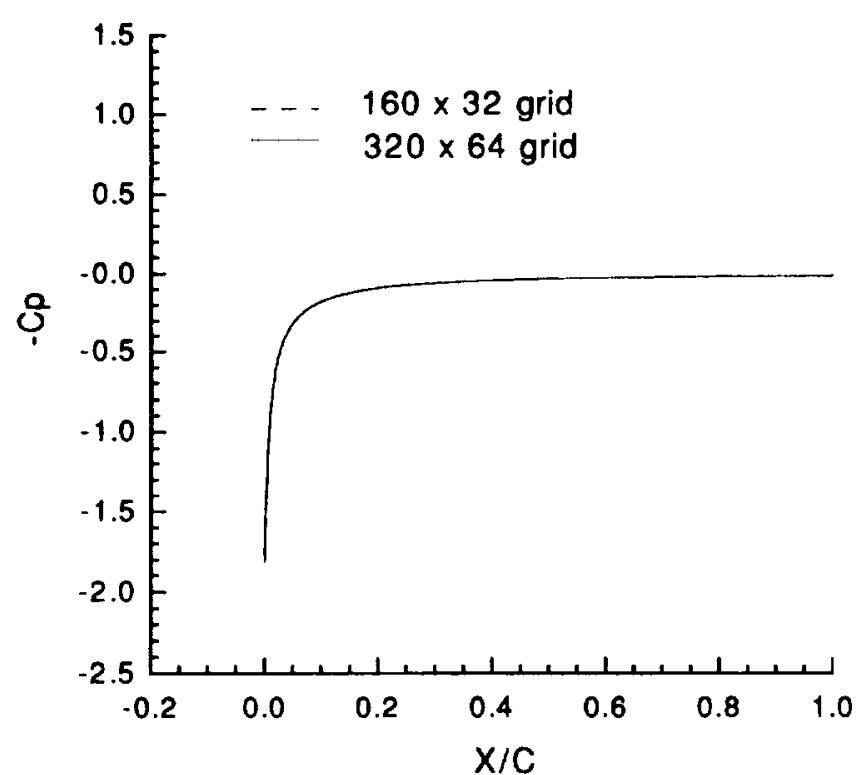

(a) Surface pressure coefficients

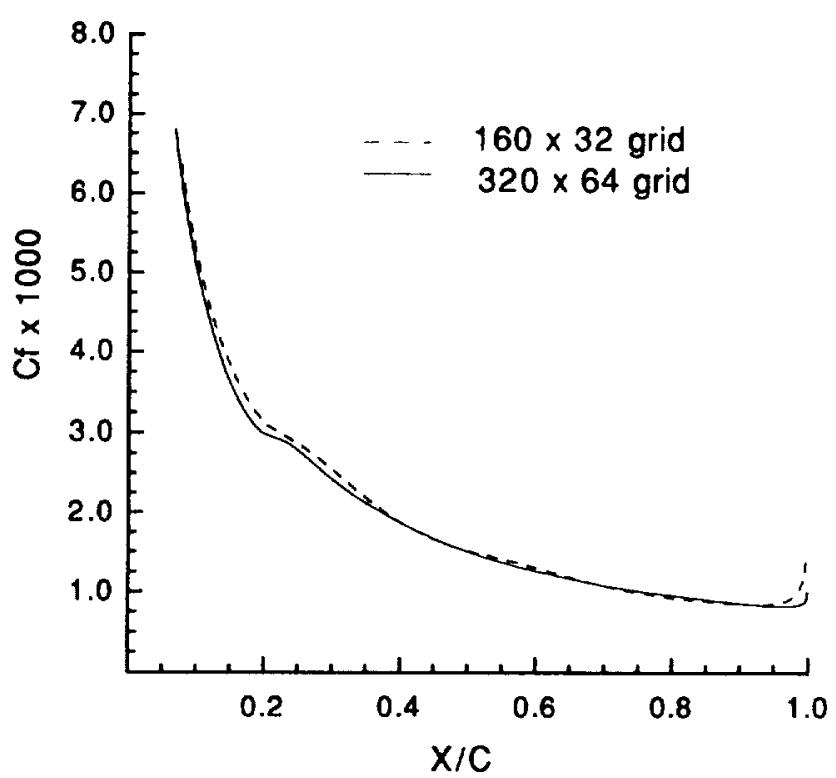

(b) Surface skin-friction coefficients

Figure 10 Surface pressure and skin-friction coefficients for Mach

20 turbulent flow over NACA 0012 airfoil $\left(\alpha=0 \mathrm{deg}, R e_{c}=10^{7}\right)$

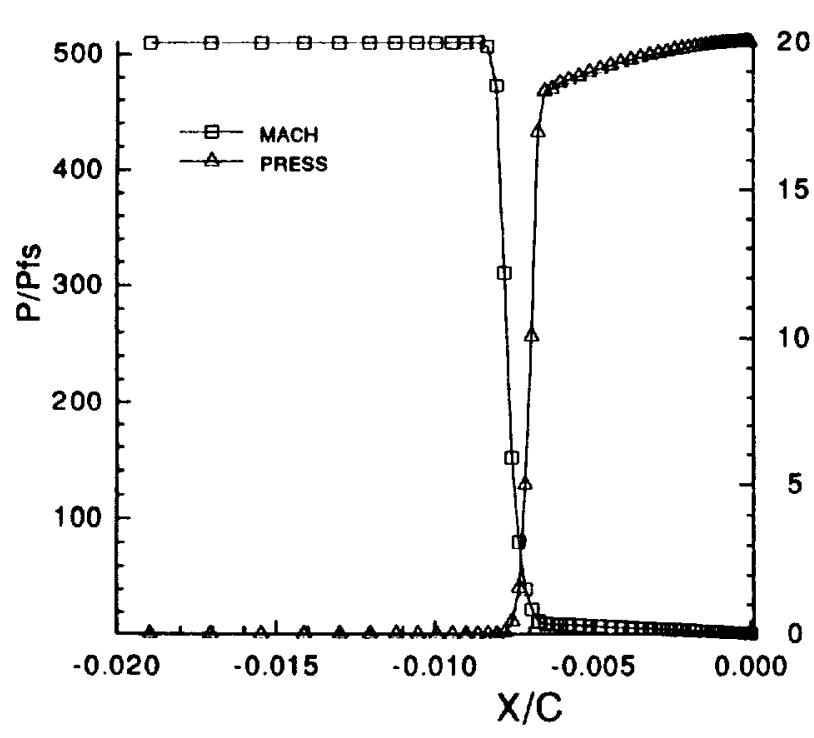

Figure 11 Pressure and Mach number variations along stagnation streamline for Mach 20 turbulent flow (NACA 0012 airfoil, $\alpha=0 \mathrm{deg}, R e_{c}=10^{7}$ )

20

15

10

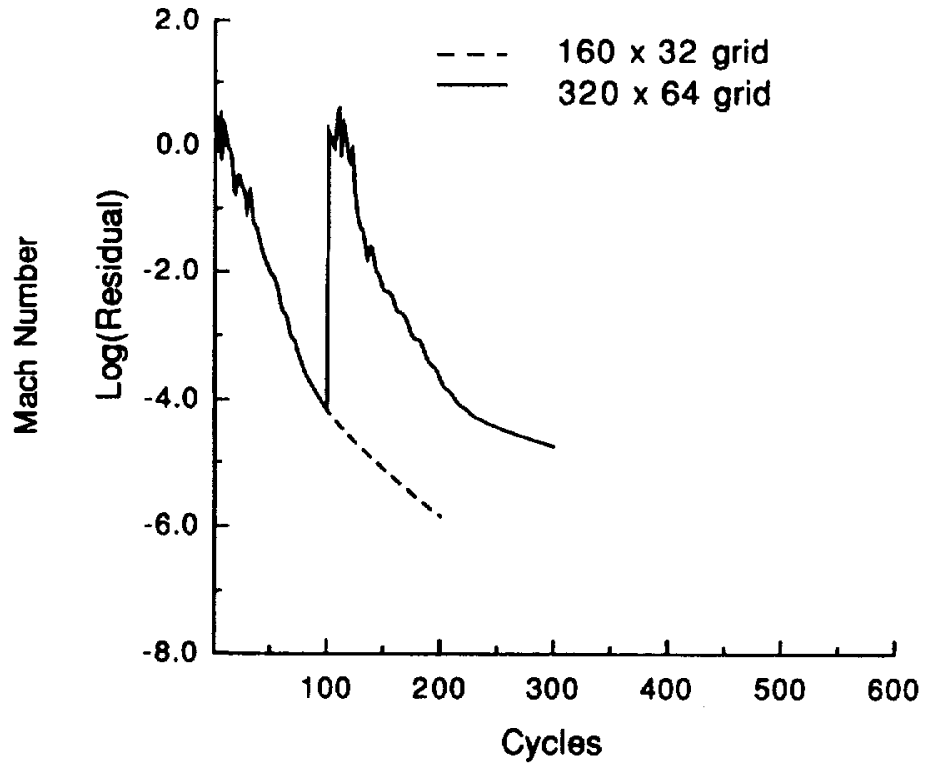

Figure 12 Convergence histories for Mach 20 turbulent flow (NACA 0012 airfoil, $\alpha=0 \mathrm{deg}, R e_{c}=10^{7}$ ) 


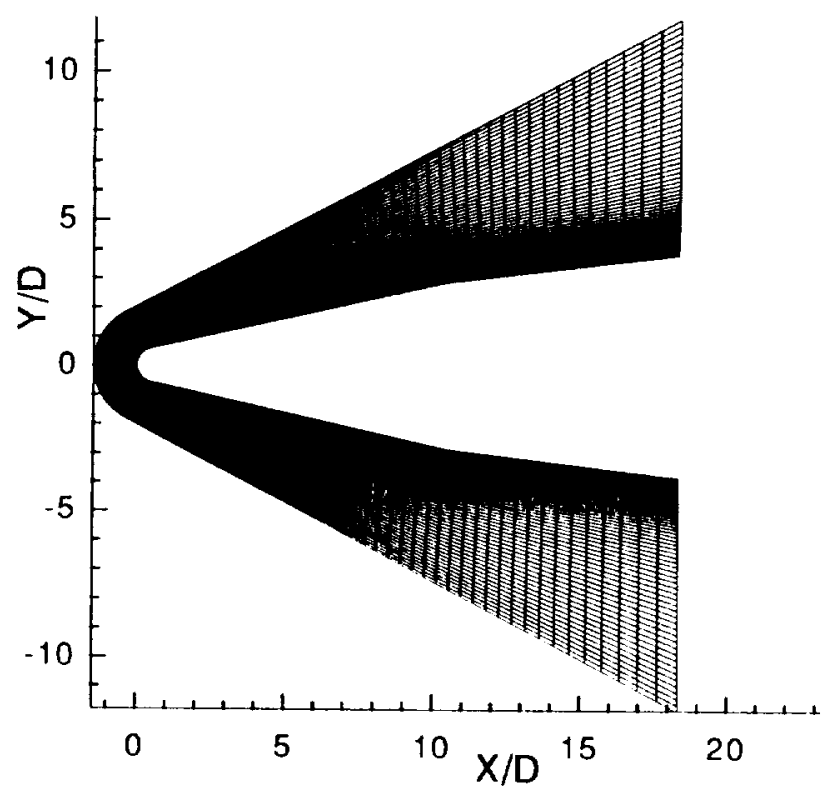

(a) Symmetry plane

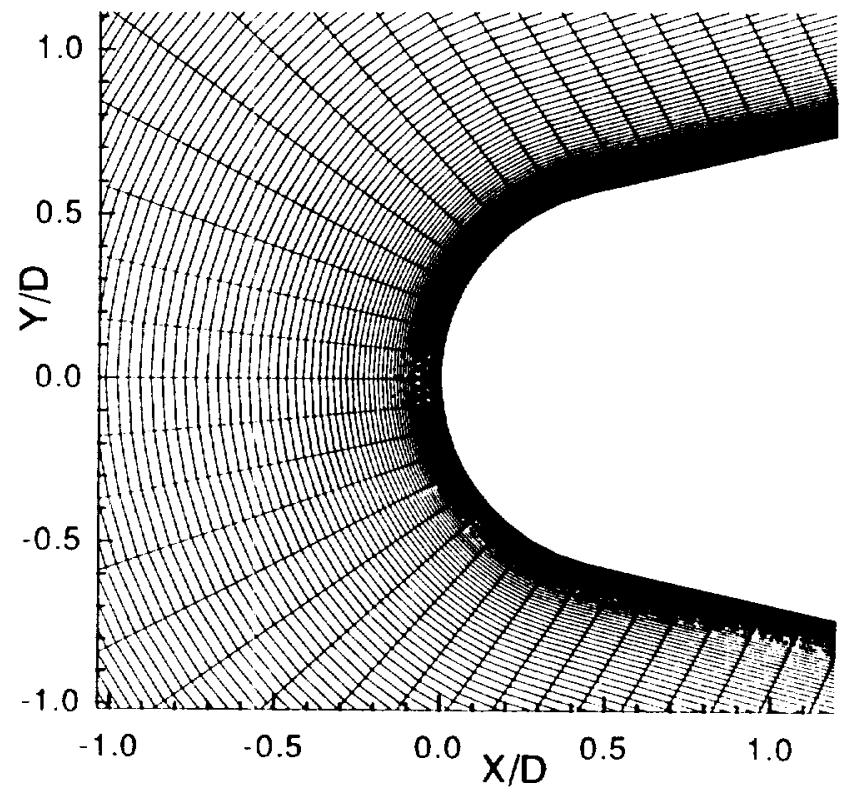

(b) Blowup of nose region

Figure 13 Grid for turbulent flow over blunt biconic $(128 \times 96 \times 24)$

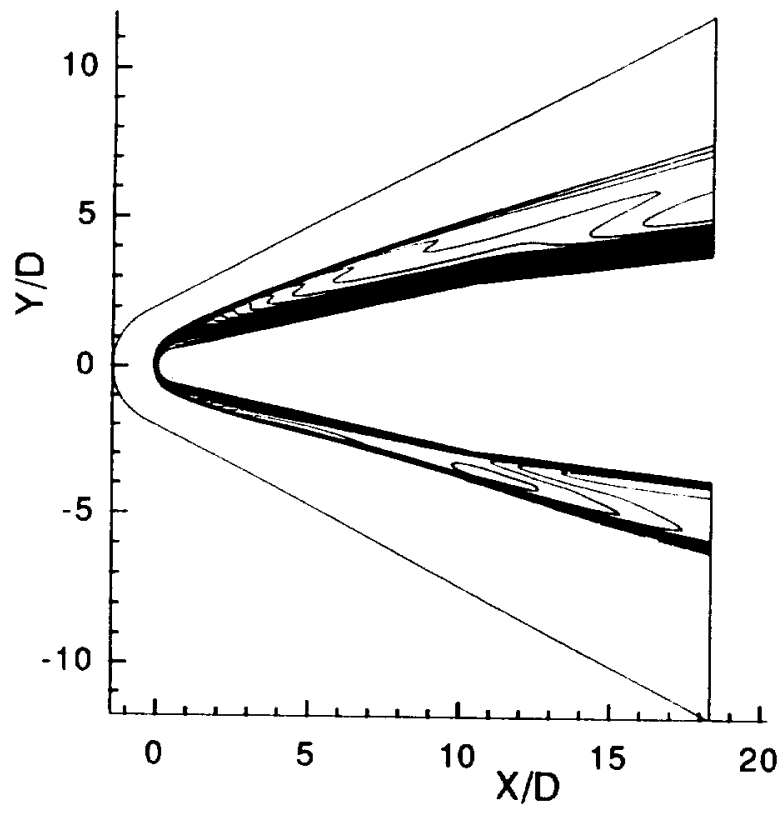

(a) Symmetry plane

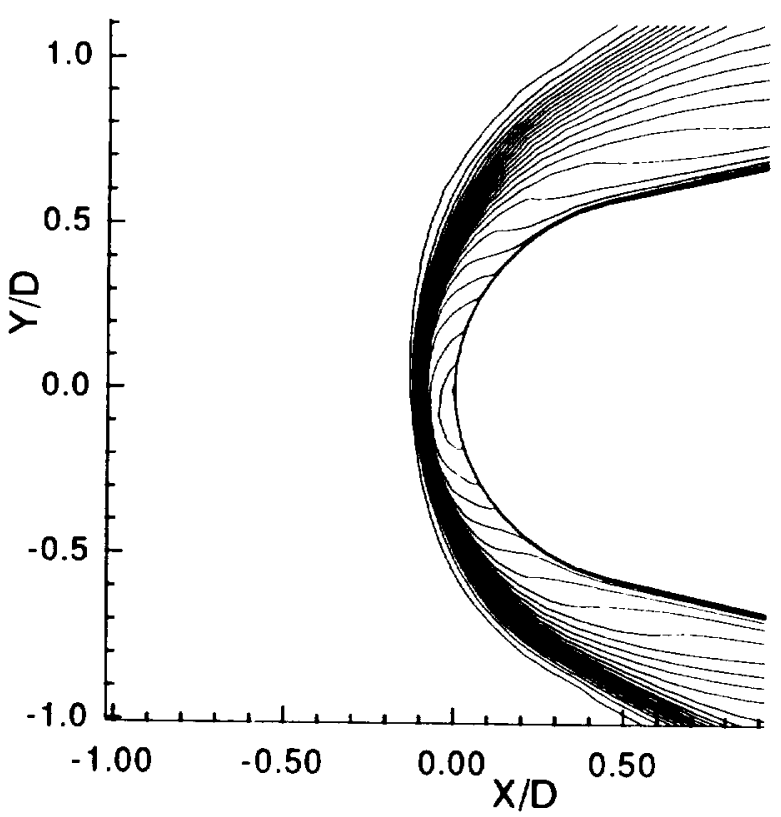

(b) Blowup of nose region

Figure 14 Mach number contours for Mach 6 turbulent flow over blunt biconic ( $\alpha=5 \mathrm{deg}, \operatorname{Re}_{D}=2.89 \times 10^{5}$ ) 


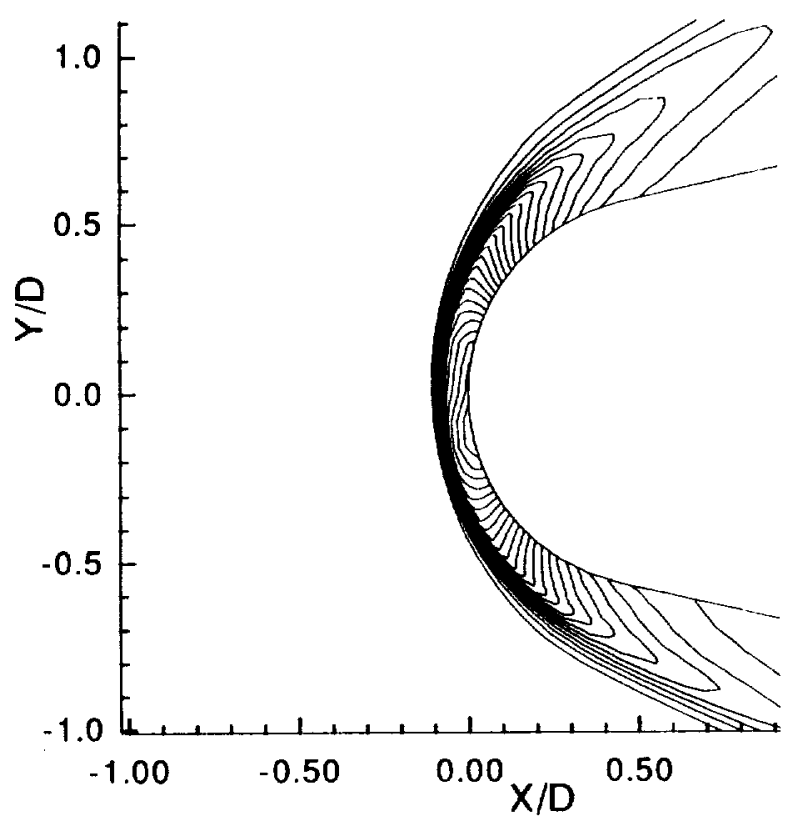

(a) Blowup of nose region

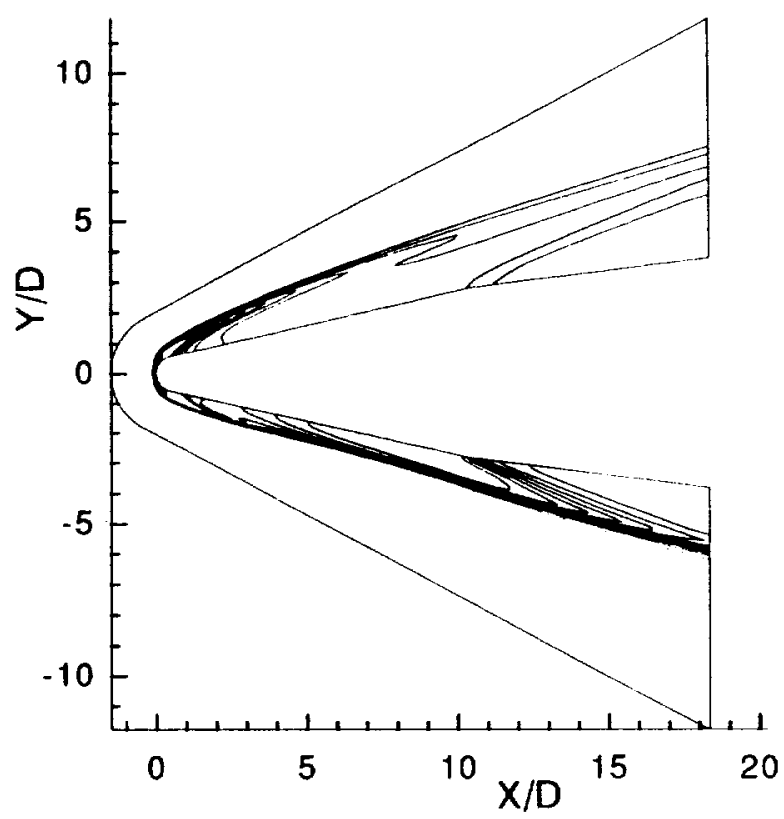

(b) Symmetry plane

Figure 15 Pressure contours for Mach 6 turbulent flow over blunt biconic $\left(\alpha=5 \mathrm{deg}, \operatorname{Re}_{D}=2.89 \times 10^{5}\right)$

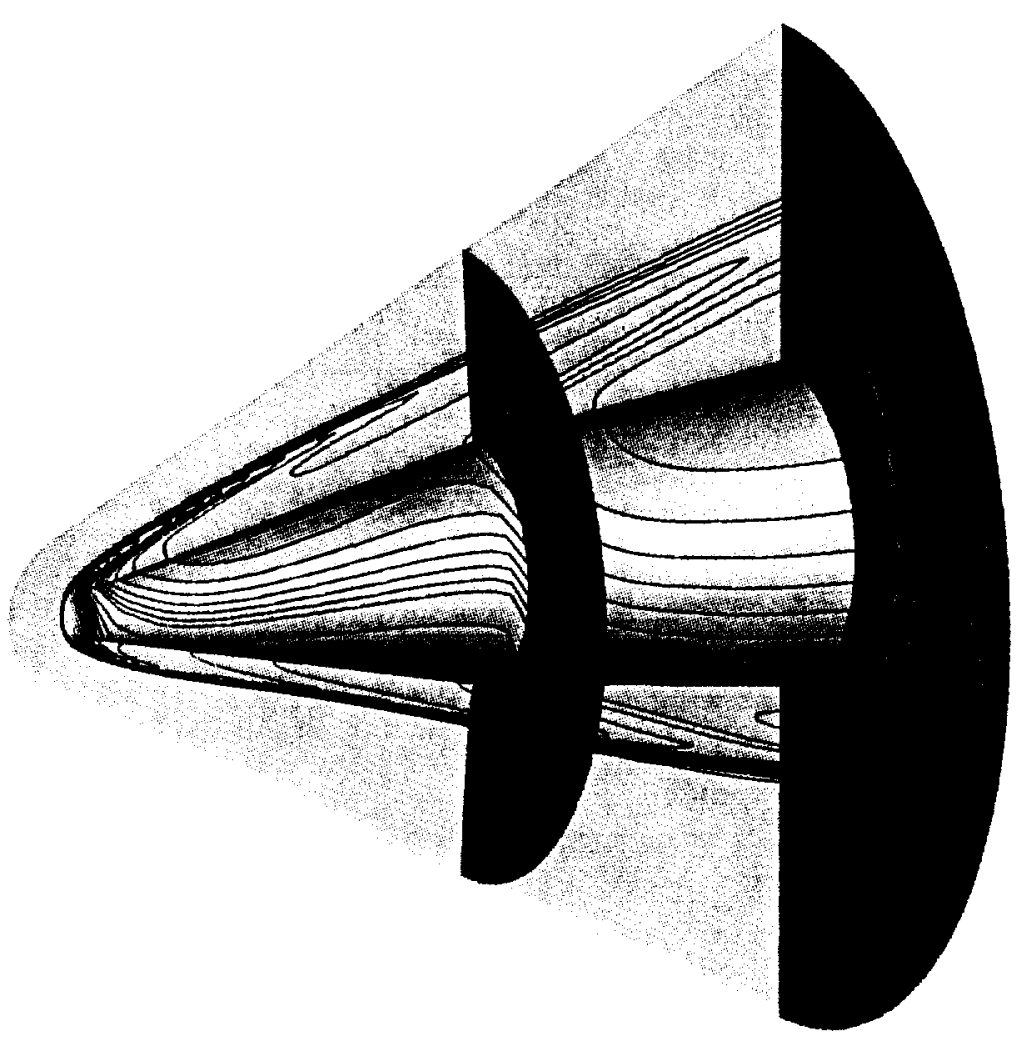

(c) Three-dimensional view 


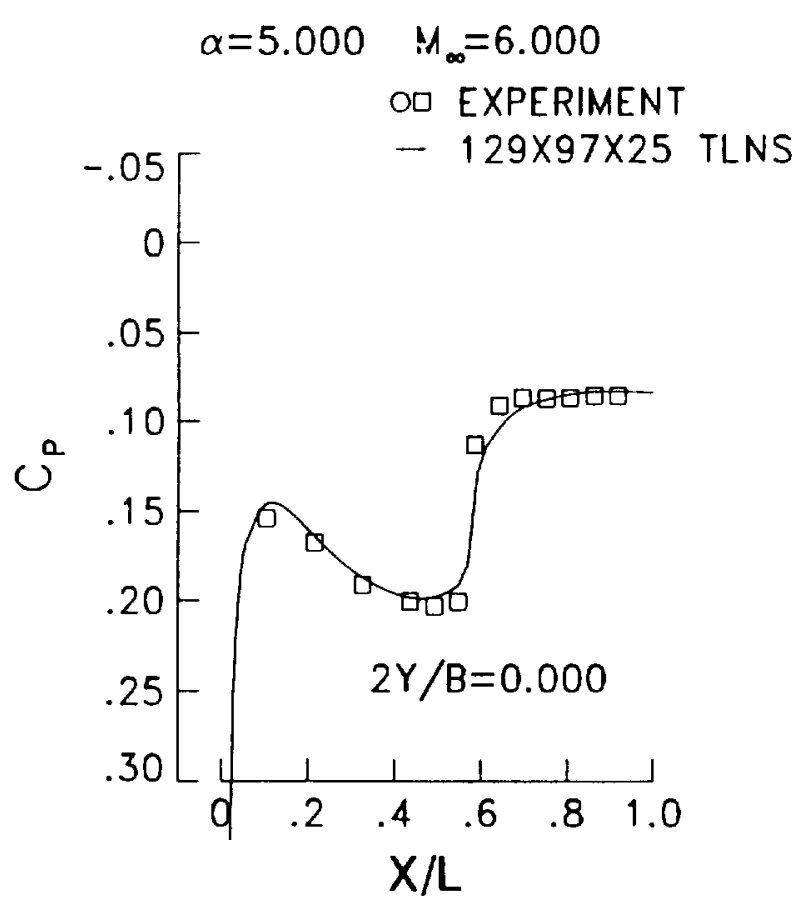

(a) Windward plane

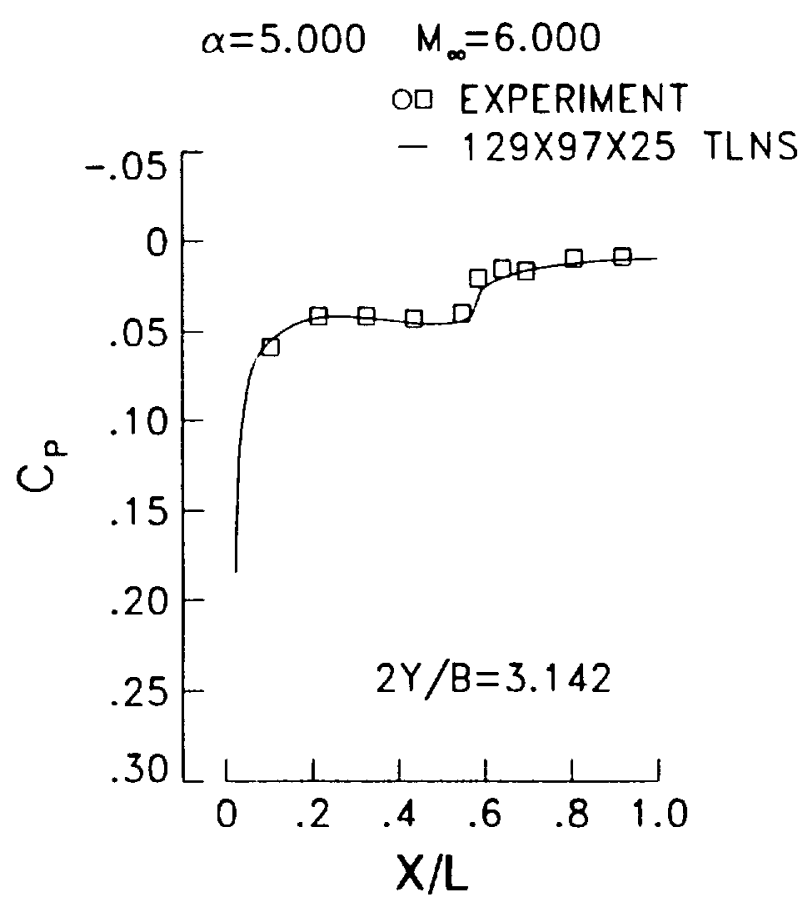

(b) Leeward plane

Figure 16 Pressure coefficient distributions in axial direction for Mach 6 turbulent flow over blunt biconic $\left(\alpha=5 \mathrm{deg}, \operatorname{Re}_{D}=2.89 \times 10^{5}\right)$

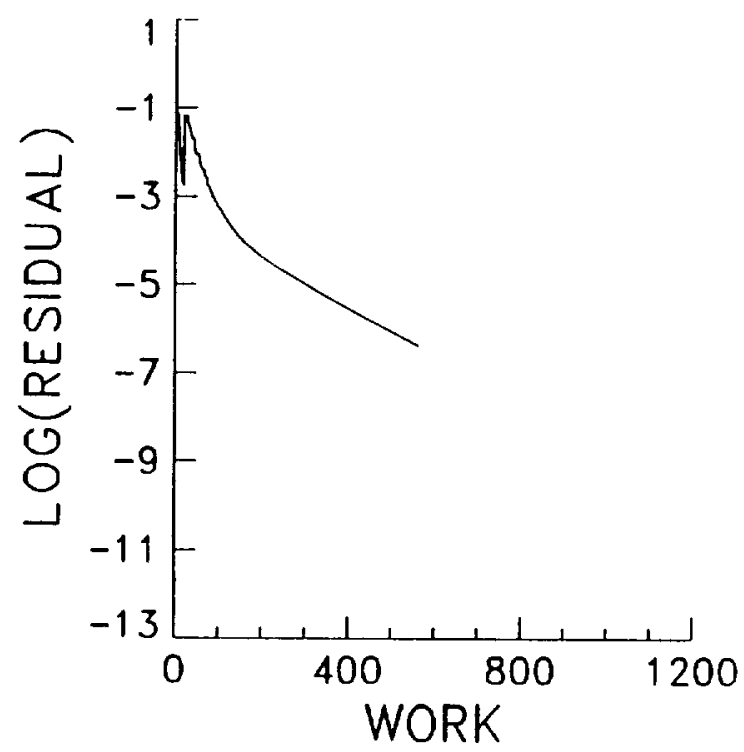

Figure 17 Convergence history (blunt biconic, $\alpha=5 \mathrm{deg}, \operatorname{Re}_{D}=2.89 \times 10^{5}$ ) 


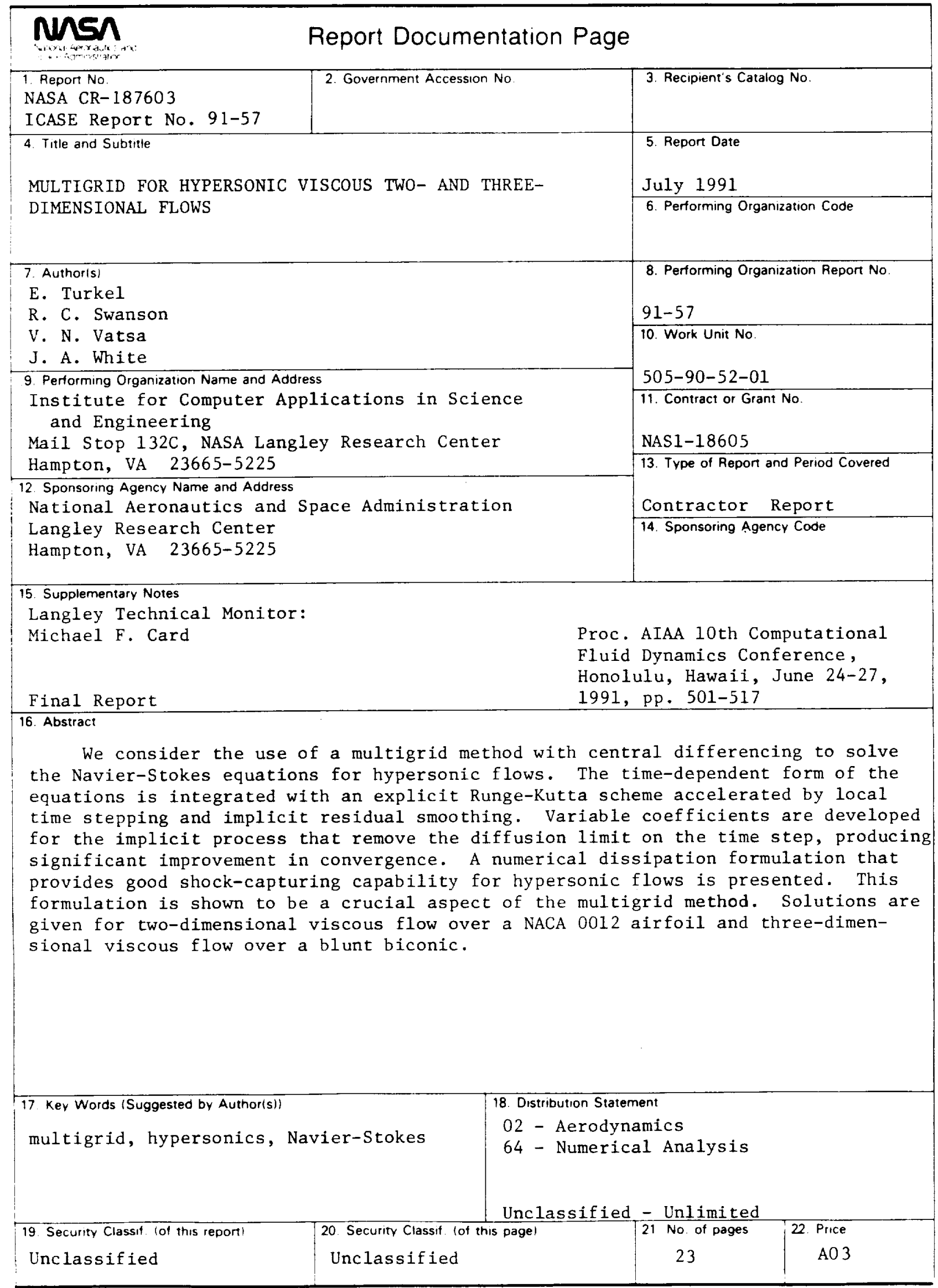

